\title{
Synchronization of Identical Oscillators Coupled through a Symmetric Network with Dynamics: A Constructive Approach with Applications to Parallel Operation of Inverters
}

\author{
Leonardo A. B. Tôrres, Member, IEEE, João P. Hespanha, Fellow, IEEE, and Jeff Moehlis
}

\begin{abstract}
We consider the problem of synchronizing a group of $N$ identical oscillators, coupled by a symmetric network that is modelled by a multiple-input/multiple-output dynamical system. We provide results that can be used to establish asymptotic synchronization of a given system and also to construct identical feedback oscillators for which synchronization is guaranteed. These results are based on a new notion of passivity with respect to manifolds defined in the input and output spaces of a dynamical system. The problem under consideration is motivated by the design of control algorithms for the parallel operation of power inverters. Simulation results have shown that synchronization could be achieved in less than two AC cycles, depending on the electrical interconnection network.
\end{abstract}

Index Terms-Synchronization, coupled oscillators, LTI network, voltage power supplies.

\section{INTRODUCTION}

This paper addresses the synchronization of identical oscillators connected through a network represented by a dynamical system as shown in Figure 1. A key motivation for this

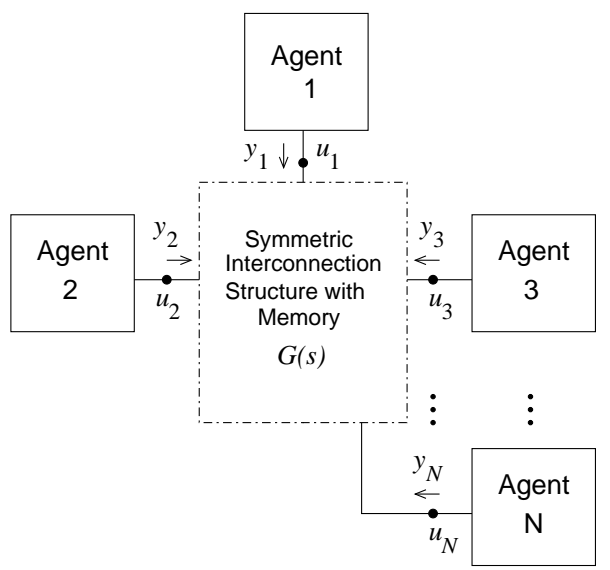

Figure 1. A group of identical nonlinear oscillators (denoted by agents) are connected through a dynamic network. Our goal is to design the agent dynamics to ensure their synchronization.

problem is the synchronization of alternative-energy power

Leonardo Tôrres is with the Department of Electronic Engineering, Universidade Federal de Minas Gerais, Belo Horizonte, CEP 31270-901, Brazil, e-mail: leotorres@ufmg.br.

João P. Hespanha and Jeff Moehlis are with the Center for Control, Dynamical Systems, and Computation, University of California, Santa Barbara, CA 93106, USA.

Manuscript received September 5, 2013; revised XXX, 2013. generators connected to a local power grid in an isolated community $[1,2]$, or the synchronization of multiple inverters providing energy to the same load [3]. We are interested in very fast synchronization (within less than 10 cycles) for which a standard phasor-domain analysis is not appropriate since non-sinusoidal signals can dominate the transient behavior (see [4] for an alternative approach). Fast synchronization is possible when power electronic devices (typically inverters) are used to interface primary energy sources with the power bus, i.e. the inverters operate in the grid-forming mode [5]. A key challenge introduced by AC power supplies with fast dynamics is that an interconnection electrical network containing inductive and/or capacitive components cannot be regarded as simply enforcing algebraic constraints between currents and voltages (in the phasor domain) and, instead, must be treated as a dynamical system, as recently pointed out in [6]. It is worth mentioning that most previous work on stability analysis of power systems using standard phasor based models neglects conductances since they are very difficult to handle mathematically, but this simplification is not made in the present paper.

With respect to previous work on parallel operation of power inverters, specifically in the contexts of Uninterruptible Power Supplies (UPS) and islanded microgrids, the Droop Method proposed in [7] is a well-known approach (see [8] and references therein). This method essentially relies on systems designed to mimic the behavior of simplified electromechanical power generators to achieve equal sharing of active and reactive power, based on continuous changes in frequency and amplitude of generated sinusoidal signals, using only variables that can be locally measured. In [9] and [10] the authors have addressed the local and regional stability analysis, respectively, associated with the use of the Droop Method. This analysis makes use of average dynamical models that are necessary to justify the assumption of almost constant interconnection impedances between parallel connected inverters (admittances shown in Figure 2). The analysis provided in the present paper is global and does not rely on the assumption of almost constant impedances, i.e. it is developed in the time domain, which makes it potentially applicable for much faster time scales. We shall see in a numerical example in Section IV that even when starting with very different phases, the power supplies synchronize in less than two AC cycles, depending on the electrical interconnection network. 
Inspired by the work of [11-13] we use dissipation and passivity [14] as key analysis tools. The use of passivity is attractive because it allows one to establish passivity properties for a large network based on input-output properties of individual components. In the context of electrical networks, passivity is also natural $[15,16]$ because an electrical network comprised only of passive elements is a passive system [17]. However, our work differs from previous passivity-based approaches to synchronization in that it is based on a new notion of passivity with respect to manifolds defined on the system's input and output spaces.

Consider a square system with $m$ inputs and $m$ outputs and two manifolds $\mathcal{U} \subset \mathbb{R}^{m}$ and $\mathcal{Y} \subset \mathbb{R}^{m}$. We define a system to be passive with respect to the pair of manifolds $(\mathcal{U}, \mathcal{Y})$ if for every system trajectory there exists another trajectory with inputs and outputs in $\mathcal{U}$ and $\mathcal{Y}$, respectively, such that the difference between the two trajectories satisfy an appropriate dissipation inequality defined precisely in Section II. For the trivial manifolds $\mathcal{U}=\mathcal{Y}=\{0\}$, this definition coincides with the usual definition of passivity [18, Definition 6.3]. However, that is not the case for other manifolds, even when applied to linear systems. This new notion of passivity was motivated by the incremental passivity approach introduced by Stan and Sepulchre in [11], but it is strictly weaker (even for linear systems) and, in fact, the gap between these notions is crucial for the results presented in this paper. In addition, several results are provided to establish the passivity of linear systems with respect to input/output linear subspaces and of several forms of composition of nonlinear systems (including feedback interconnections, diagonal compositions, and parallel interconnections).

The synchronization results can be found in Section III, which considers a system consisting of $N$ feedback oscillators connected by a symmetric network (see Figure 1). Two key differences with respect to previous work on the general problem of oscillators' synchronization are that the interconnection network is assumed to have dynamics and that the coupling between the oscillators persists even when they become synchronized. In most of the work on synchronization of dynamical agents the coupling between agents takes place through "error" signals that vanish when the system becomes synchronized. In those works, the coupling is "transient" in the sense that the interaction between agents disappears asymptotically as they become synchronized. Moreover, these error signals that drive the agents towards consensus are static functions of the difference between variables associated with "neighboring" agents [19]. Instead, here the agents are coupled through their connection to a multiple-input/multipleoutput dynamical system and continue to interact with this interconnection structure and with each other even when they are synchronized. This type of topology is needed to study the interconnection of electric power supplies to a common load [20], as shown in Figure 2. In this contribution we offer a theoretical basis for a deeper comprehension of the adhoc procedures outlined in [20], and experimentally tested in [21]. However, it is important to notice that our aim here, different from the objectives in [20, 21], is to investigate synchronization conditions related to agents' consensus without specifying a target oscillation amplitude, frequency or shape (e.g. sinusoidal) for the agents' outputs after reaching the synchronized behavior.

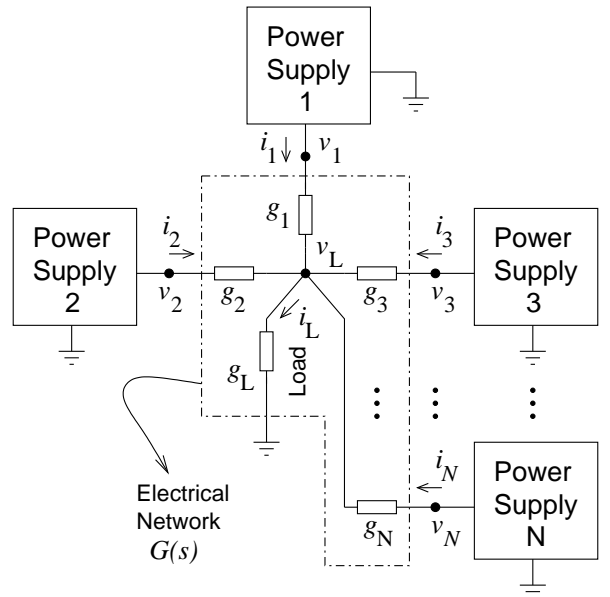

Figure 2. Interconnection of $N$ voltage sources that provide energy to a common load using a star configuration. The network that interconnects the power supplies contains one or more loads, corresponding to the admittance $g_{\mathrm{L}}$, and several connecting components, corresponding to the admittances $g_{1}$ through $g_{N}$. This network can be modeled by a transfer function matrix $G(s)$ from the vector of voltages $\left[v_{1}, v_{2}, \ldots, v_{n}\right]$ to the vector of currents $\left[i_{1}, i_{2}, \ldots, i_{n}\right]$.

The main synchronization result in Section III is a set of sufficient conditions for the synchronization of $N$ identical feedback oscillators connected by a symmetric dynamic network. Our constructive approach means that these conditions are amenable to the design of the oscillatory agents that guarantee the synchronization of the overall system regardless of the initial conditions of the oscillators and the network that couples them. In fact, a systematic procedure is provided in Sections III-C-III-E for the construction of oscillators, with closed form solutions provided for star networks with an arbitrary number of agents, resistive interconnection branches, and RLC oscillator dynamics.

\section{PASSIVITY WITH RESPECT TO MANIFOLDS}

In this section we develop a passivity-inspired approach for the study of networks of feedback oscillators. To this effect, consider a dynamical system with the same number of inputs and outputs of the form

$$
\dot{x}=f(x, u), \quad y=g(x, u), \quad x \in \mathbb{R}^{n}, u, y \in \mathbb{R}^{m} .
$$

Throughout this paper we assume solutions to ODEs in the sense of Carathéodory [22]. We now introduce definitions that express properties of the difference between arbitrary solutions to (1) and solutions whose inputs and outputs lie in specific manifolds $\mathcal{U}, \mathcal{Y} \subset \mathbb{R}^{m}$. We recall that a projection onto a manifold $\mathcal{M} \subset \mathbb{R}^{m}$ is a function $\pi: \mathbb{R}^{m} \rightarrow \mathcal{M}$ for which $\pi(z)=z, \forall z \in \mathcal{M}$, and $\pi(\pi(x))=\pi(x), \forall x \in \mathbb{R}^{m}$.

We say that (1) is (non-strictly) passive with respect to the pair $(\mathcal{U}, \mathcal{Y})$ if there exists a continuously differentiable, positive definite storage function $S: \mathbb{R}^{n} \rightarrow[0, \infty)$ and a projection $\pi$ of $\mathbb{R}^{m}$ onto $\mathcal{U}$, such that for every globally defined solution $(u, x, y)$ to (1) there exists another globally defined 
solution $(\bar{u}, \bar{x}, \bar{y})$ to the same system (possibly corresponding to a different initial condition) with $\bar{u}(t)=\pi(u(t))$ and $\bar{y}(t) \in \mathcal{Y}, \forall t \geqslant 0$ for which

$$
\dot{s}(t) \leqslant(y(t)-\bar{y}(t))^{\prime}(u(t)-\bar{u}(t)), \quad \forall t \geqslant 0 ;
$$

where $s(t):=S(x(t)-\bar{x}(t)), \forall t \geqslant 0$, and $\dot{s}(t)$ denotes the time-derivative of $s(t)$.

Following a terminology consistent with that of [18, p. 236], and defining $\tilde{u}(t)=u(t)-\bar{u}(t), \tilde{y}(t)=y(t)-\bar{y}(t)$, with $\bar{u}(t) \in \mathcal{U}$ and $\bar{y}(t) \in \mathcal{Y}, \forall t \geqslant 0$, we introduce the following additional notions of passivity with respect to manifolds: We say that (1) is input-feedforward passive with respect to $(\mathcal{U}, \mathcal{Y})$ with passivity margin $\sigma \in \mathbb{R}$ if

$$
\dot{s}(t) \leqslant-2 \sigma\|\tilde{u}(t)\|^{2}+(\tilde{y}(t))^{\prime}(\tilde{u}(t)), \quad \forall t \geqslant 0,
$$

holds instead of (2a), and when $\sigma>0$ the system is called input-strictly passive with respect to $(\mathcal{U}, \mathcal{Y})$. We say that $(1)$ is output-feedback passive with respect to $(\mathcal{U}, \mathcal{Y})$ with passivity margin $\sigma \in \mathbb{R}$ if

$$
\dot{s}(t) \leqslant-2 \sigma\|\tilde{y}(t)\|^{2}+(\tilde{y}(t))^{\prime}(\tilde{u}(t)), \quad \forall t \geqslant 0,
$$

holds instead of (2a), and when $\sigma>0$ we say that (1) is output-strictly passive with respect to $(\mathcal{U}, \mathcal{Y})$. We say that $(1)$ is strictly passive with respect to $(\mathcal{U}, \mathcal{Y})$ with passivity margin $\sigma \in \mathbb{R}, \sigma>0$, if

$$
\dot{s}(t) \leqslant-2 \sigma s(t)+(\tilde{y}(t))^{\prime}(\tilde{u}(t)), \quad \forall t \geqslant 0 .
$$

holds instead of (2a). These definitions extend to memoryless systems, simply by making $s(t)=\dot{s}(t)=0$ in the above inequalities, and by disregarding the need to choose appropriately initial conditions. In addition, for trivial manifolds $\mathcal{U}=\mathcal{Y}=\{0\}$ (for which, $\bar{u}=\bar{y}=0$ ) the definitions above coincide with the notions of passivity in [18, Definition 6.3] (specialized for $\varphi$, in [18], equal to the identity function).

\section{A. Input/output convergence to manifolds}

Consider the positive feedback interconnection of (1) with a static nonlinearity of the form

$$
u(t)=\Phi(y(t)), \quad \forall t \geqslant 0 .
$$

For the closed-loop system (1), (3) to be well-defined, we assume that for every $x \in \mathbb{R}^{n}$, the equation $y=g(x, \Phi(y))$ has a unique solution $y:=h(x)$ so that the closed-loop dynamics are given by $\dot{x}=f(x, \Phi(h(x)))$, and the map $x \mapsto f(x, \Phi(h(x)))$ is locally Lipschitz continuous.

Our general goal is to determine if the signals $u(t)$ and $y(t)$ converge to manifolds $\mathcal{U}$ and $\mathcal{Y}$, respectively, along solutions to (1), (3). For some specific manifolds - e.g., the linear space of all vectors in $\mathbb{R}^{m}$ with equal entries - this convergence necessarily implies synchronization of the inputs and/or outputs, in the sense that all the inputs become equal and/or all outputs become equal. This goal is closely related to more common notions of invariance and attractiveness of synchronization manifolds [23], but here the manifolds are defined in the input and output spaces in $\mathbb{R}^{m}$, instead of being defined in the system's state space in $\mathbb{R}^{n}$.
Theorem 1 (Input/output convergence). Any one of the following conditions guarantees that $u(t)$ converges to $\mathcal{U}$ :

C1 Suppose that (1) is strictly passive with respect to the pair $(\mathcal{U}, \mathcal{Y})$. This implies that for every globally defined solution $(u, x, y)$ to (1), (3) there exists globally defined solutions $(\bar{u}, \bar{x}, \bar{y})$ to $(1)$ with $\bar{u}(t)=\pi(u(t)), \bar{y}(t) \in \mathcal{Y}$, $\forall t \geqslant 0$ such that $(2 \mathrm{~d})$ holds. Assume that among such solutions there exists one for which

$$
\left[\delta_{\Phi, \pi}(y(t))\right]^{\prime}[\tilde{y}(t)] \leqslant 0,
$$

with $\delta_{\Phi, \pi}(y(t)):=\Phi(y(t))-(\pi \circ \Phi)(y(t))$ and $\tilde{y}(t):=$ $y(t)-\bar{y}(t), \forall t \geqslant 0$. Then $u(t)$ converges to $\mathcal{U}$ and $y(t)$ converges to $\mathcal{Y}$ as $t \rightarrow \infty$, along every solution to (1), (3) that is globally defined.

C2 Suppose that (1) is input-feedforward passive with respect to the pair $(\mathcal{U}, \mathcal{Y})$ with passivity margin $\sigma>\alpha$, for a radially unbounded storage function $S$. This implies that for every globally defined solution $(u, x, y)$ to (1), (3) there exists globally defined solutions $(\bar{u}, \bar{x}, \bar{y})$ to (1) with $\bar{u}(t)=\pi(u(t)), \bar{y}(t) \in \mathcal{Y}, \forall t \geqslant 0$ such that (2b) holds. Assume that among such solutions there exists one for which

$$
\left[\delta_{\Phi, \pi}(y(t))\right]^{\prime}[\tilde{y}(t)] \leqslant 2 \alpha\left\|\delta_{\Phi, \pi}(y(t))\right\|^{2},
$$

with $\delta_{\Phi, \pi}(y(t)):=\Phi(y(t))-(\pi \circ \Phi)(y(t))$ and $\tilde{y}(t):=$ $y(t)-\bar{y}(t), \forall t \geqslant 0$. Then $u(t)$ converges to $\mathcal{U}$ as $t \rightarrow \infty$, along every solution to (1), (3) that is globally defined and uniformly bounded solution ${ }^{1}$.

C3 Suppose that (1) is output-feedback passive with respect to the pair $(\mathcal{U}, \mathcal{Y})$ with passivity margin $\sigma>\alpha$, for a radially unbounded storage function $S$. This implies that for every globally defined solution $(u, x, y)$ to (1), (3) there exists globally defined solutions $(\bar{u}, \bar{x}, \bar{y})$ to $(1)$ with $\bar{u}(t)=\pi(u(t)), \bar{y}(t) \in \mathcal{Y}, \forall t \geqslant 0$ such that $(2 \mathrm{c})$ holds. Assume that among such solutions there exists one for which

$$
\left[\delta_{\Phi, \pi}(y(t))\right]^{\prime}[\tilde{y}(t)] \leqslant 2 \alpha\|\tilde{y}(t)\|^{2},
$$

with $\delta_{\Phi, \pi}(y(t)):=\Phi(y(t))-(\pi \circ \Phi)(y(t))$ and $\tilde{y}(t):=$ $y(t)-\bar{y}(t), \forall t \geqslant 0$. Then $u(t)$ converges to $\mathcal{U}$ and $y(t)$ converges to $\mathcal{Y}$ as $t \rightarrow \infty$, along every solution to (1), (3) that is globally defined and belongs to a uniformly bounded set of solutions.

Before proving Theorem 1, we make three observations that provide useful insights into this result and its application.

Remark 1 (Inequalities along specialized solutions). The inequalities (4a)-(4c) only need to be verified along pairs of solutions $(u, x, y),(\bar{u}, \bar{x}, \bar{y})$ for which $\bar{u}(t)=\pi(u(t))=$ $\pi \circ \Phi(y(t))$ and $\bar{y}(t) \in \mathcal{Y}, \forall t \geqslant 0$. In particular, (4a)-(4c) need not apply to arbitrary signals $y(t)$ and $\bar{y}(t)$. Notice also that, in general, $\bar{u}(t) \neq \Phi(\bar{y}(t))$. These considerations will be instrumental, e.g., in using Theorem 1 to prove Theorem 3, later in Section III-A.

\footnotetext{
${ }^{1}$ We say that a solution $x:[0, \infty) \rightarrow \mathbb{R}^{n}$ is uniformly bounded if $\|x(t)\| \leqslant$ $c<\infty$, with $c$ the same constant value $\forall t \geqslant 0$.
} 
Remark 2 (Assumption on the solutions). All three conditions in Theorem 1 only establish convergence for solutions to (1), (3) that are globally defined, so to draw conclusions that are valid for every solution, one must independently establish that every (maximal) solution is globally defined. The conditions $C 2$ and $C 3$ are even more restrictive in the sense that they only establish convergence for solutions that are bounded, which would need to be independently established. Fortunately, many powerful techniques are available to prove global existence and uniform boundedness of solutions to ODEs (cf., e.g., [18, p. 172]). For the purposes of the results in this paper, a sufficient condition is that the system (1) is input-to-state stable with respect to the input $u[18, p .172]$ and the nonlinearity $\Phi$ is bounded. This is typically the case for feedback oscillators (to be defined in Section III), where (1) would be a stable system with a strong resonance at the desired oscillation frequency and the nonlinearity $\Phi$ is used to destabilize the system, while keeping the solutions bounded.

Remark 3 (Passivity versus passivity with respect to manifolds). To conclude from Theorem 1 that $u(t)$ converges to a signal $\bar{u}(t)$ in the set $\mathcal{U}$ that is not the trivial signal $\bar{u}(t)=0, \forall t \geqslant 0$, the system (1) must be endowed with passivity properties with respect to a pair $(\mathcal{U}, \mathcal{Y})$, but not with respect to the trivial pair $(\{0\},\{0\})$. This is because the latter would imply convergence of $u(t)$ to the origin. Therefore, when we want to use Theorem 1 to establish convergence to non-zero solutions, we must rely on the "gap" between passivity with respect to appropriate manifolds $(\mathcal{U}, \mathcal{Y})$ and the standard (more conservative) notion of passivity, which would correspond to passivity with respect to the trivial manifolds $(\{0\},\{0\})$.

Remark 4 (Incremental Passivity versus passivity with respect to manifolds). The notions above are also related to the concept of Incremental Passivity in [11, 24], which would require the inequalities in (2) to hold for every pair of solutions $(u, x, y),(\bar{u}, \bar{x}, \bar{y})$ to $(1)$, and not just for the ones associated with $\bar{u}=\pi(u) \Rightarrow \bar{u} \in \mathcal{U}$, with $\bar{y} \in \mathcal{Y}$ by appropriately choosing initial conditions. The incremental passivity property can also be used to derive sufficient conditions to show that any solution to (1) will converge to a specific one, e.g. a solution such that $y(t)=0, \forall t \geqslant 0$, as it was done in [24]. However the incremental passivity property is strictly stronger than the passivity with respect to manifolds since an incrementally passive system is actually a passive system with respect to the pair $\left(\mathcal{U} \equiv \mathbb{R}^{m}, \mathcal{Y} \equiv \mathbb{R}^{m}\right)$. By restricting the pairs of solutions for which the inequalities must hold, we enlarge the class of systems that are incrementally passive with respect to nontrivial input and output manifolds.

Proof of Theorem 1. Assuming that (1) is strictly passive with respect to the pair $(\mathcal{U}, \mathcal{Y})$, for every globally defined solution $(u, x, y)$ to the feedback interconnection (1), (3), there exists another globally defined solution $(\bar{u}, \bar{x}, \bar{y})$ to (1) for which $\bar{u}(t)=\pi(u(t))$, with $\bar{y}(t) \in \mathcal{Y}, \forall t \geqslant 0$, and

$$
\dot{s} \leqslant-2 \sigma s+(\tilde{y})^{\prime}(\tilde{u})=-2 \sigma s+(\tilde{y})^{\prime}\left(\delta_{\Phi, \pi}(y)\right) \leqslant-2 \sigma s,
$$

where $s(t):=S(x(t)-\bar{x}(t))$ for a positive definite storage function $S: \mathbb{R}^{n} \rightarrow[0, \infty)$, and the second inequality is a consequence of (4a) by noticing that $\bar{u}=(\pi \circ \Phi)(y)$. Using the Comparison Lemma [18, p. 102] we conclude that $s(t)$ converges to zero exponentially fast and therefore the solution $(u, x, y)$ converges to the solution $(\bar{u}, \bar{x}, \bar{y})$ as $t \rightarrow \infty$. When (1) is input-feedforward passive with respect to the pair $(\mathcal{U}, \mathcal{Y})$ with passivity margin $\sigma>\alpha$, for every bounded and globally defined solution $(u, x, y)$ to the feedback interconnection (1), (3), we now conclude, from the definition of passivity with respect to $(\mathcal{U}, \mathcal{Y})$, that there exists another globally defined solution $(\bar{u}, \bar{x}, \bar{y})$ to (1) for which $\bar{u}(t)=\pi(u(t))$, with $\bar{y}(t) \in$ $\mathcal{Y}, \forall t \geqslant 0$, and

$$
\begin{aligned}
\dot{s} & \leqslant-2 \sigma\|\Phi(y)-\bar{u}\|^{2}+(\tilde{y})^{\prime}(\Phi(y)-\bar{u}), \\
& \leqslant-2 \sigma\left\|\delta_{\Phi, \pi}(y)\right\|^{2}+(\tilde{y})^{\prime}\left(\delta_{\Phi, \pi}(y)\right), \\
& \leqslant-2(\sigma-\alpha)\left\|\delta_{\Phi, \pi}(h(x))\right\|^{2}
\end{aligned}
$$

where $s(t):=S(x(t)-\bar{x}(t))$ for a positive definite and radially unbounded storage function $S: \mathbb{R}^{n} \rightarrow[0, \infty)$, the last inequality is a consequence of (4b), and the function $y=h(x)$ is associated with the unique solution to $y=g(x, \Phi(y))$. Moreover, the state vector $\bar{x}$ must be bounded because from the above inequality $s$ is bounded, and by assumption $S$ is radially unbounded and $x$ belongs to a uniformly bounded set of solutions. Using LaSalle's invariance principle [25, Theorem 6.4] to the combined dynamics of $x$ and $\bar{x}$, we conclude that $(x(t), \bar{x}(t)) \in \mathbb{R}^{2 n}, \forall t \geqslant 0$ must converge to the largest invariant set $M$ contained in

$$
E:=\left\{(x, \bar{x}) \in \mathbb{R}^{2 n}: \Phi(h(x))=\pi \circ \Phi(h(x))\right\} .
$$

Since for any pair of states $(x(t), \bar{x}(t))$ in $E$ (and in $M$ ) we have $u(t)=\bar{u}(t)$, we conclude that $\tilde{u}(t)=u(t)-\bar{u}(t)$ converges to 0 as $t \rightarrow \infty$ and therefore $u(t)$ must converge to $\mathcal{U}$. Assuming that (1) is output-strictly passive with respect to the pair $(\mathcal{U}, \mathcal{Y})$, for every bounded and globally defined solution $(u, x, y)$ to the feedback interconnection (1), (3), we now conclude that there exists a solution $(\bar{u}, \bar{x}, \bar{y})$ to (1) for which $\bar{u}(t)=\pi(u(t))$, with $\bar{y}(t) \in \mathcal{Y}, \forall t \geqslant 0$, and

$$
\begin{aligned}
\dot{s} & \leqslant-2 \sigma\|\tilde{y}\|^{2}+(\tilde{y})^{\prime}(\Phi(y)-\bar{u}), \\
& \leqslant-2 \sigma\|\tilde{y}\|^{2}+(\tilde{y})^{\prime}\left(\delta_{\Phi, \pi}(y)\right), \\
& \leqslant-2(\sigma-\alpha)\|\tilde{y}\|^{2}=-2(\sigma-\alpha)\|h(x)-h(\bar{x})\|,
\end{aligned}
$$

where $s(t):=S(x(t)-\bar{x}(t))$ for a positive definite and radially unbounded storage function $S: \mathbb{R}^{n} \rightarrow[0, \infty)$, and the last inequality is a consequence of (4c). Reasoning as above, we now conclude using LaSalle's invariance principle that $y(t)$ converges to $\bar{y}(t)$ as $t \rightarrow \infty$ and therefore $y(t)$ converges to $\mathcal{Y}$. From (3), we further conclude that $u(t)$ converges to $\bar{u}(t)$ as $t \rightarrow \infty$ and therefore $u(t)$ must converge to $\mathcal{U}$.

\section{B. Linear case}

Consider now the specific case where (1) is a linear timeinvariant (LTI) system of the form

$$
\dot{x}=A x+B u, \quad y=C x+D u, \quad x \in \mathbb{R}^{n}, u, y \in \mathbb{R}^{m},
$$


and two manifolds $\mathcal{U}$ and $\mathcal{Y}$ that are linear subspaces of $\mathbb{R}^{m}$ defined by appropriate $m \times m$ projection matrices $U=U^{2}$ and $Y=Y^{2}$, respectively, such that $\mathcal{U}=\operatorname{Im} U$ and $\mathcal{Y}=\operatorname{Im} Y$.

We say that a pair of matrices $U, Y \in \mathbb{R}^{m \times m}$ commutes with the system (5) when

$$
\hat{G}(s) U=Y \hat{G}(s), \quad \forall s \in \mathbb{C} .
$$

where $\hat{G}(s):=C(s I-A)^{-1} B+D$. As a consequence of (6), the LTI system Markov parameters and the impulse response are such that

$$
\begin{aligned}
& D U=Y D, C A^{k} B U=Y C A^{k} B, \quad k=0,1, \ldots, n-1 \\
& D U=Y D, C e^{A t} B U=Y C e^{A t} B, \quad \forall t \geqslant 0 .
\end{aligned}
$$

We are now ready to establish passivity of (5) with respect to $(\mathcal{U}, \mathcal{Y})$ using linear matrix inequalities (LMIs).

Theorem 2 (LTI case). Suppose that the pair of projection matrices $U, Y \in \mathbb{R}^{m \times m}$ commutes with the system (5), and define $T:=\left[\begin{array}{cc}I_{n} & 0 \\ 0 & I_{m}-U\end{array}\right]$, where $I_{k}$ denotes the $k \times k$ identity matrix. The system (5) is passive with respect to $(\mathcal{U}:=\operatorname{Im} U, \mathcal{Y}:=\operatorname{Im} Y)$ if there exists a matrix $P \in \mathbb{R}^{n \times n}$, $P=P^{\prime}>0$, such that

$$
T^{\prime}\left[\begin{array}{cc}
P A+A^{\prime} P & P B-C^{\prime} \\
B^{\prime} P-C & -D-D^{\prime}
\end{array}\right] T \leqslant 0 ;
$$

it is strictly passive with respect to $(\mathcal{U}, \mathcal{Y})$ with dissipation margin $\sigma>0$ if there exists a matrix $P \in \mathbb{R}^{n \times n}, P=P^{\prime}>0$, such that

$$
T^{\prime}\left[\begin{array}{cc}
P A+A^{\prime} P+2 \sigma P & P B-C^{\prime} \\
B^{\prime} P-C & -D-D^{\prime}
\end{array}\right] T \leqslant 0 ;
$$

it is input-feedforward passive with respect to $(\mathcal{U}, \mathcal{Y})$ with passivity margin $\sigma \in \mathbb{R}$ if there exists a matrix $P \in \mathbb{R}^{n \times n}$, $P=P^{\prime}>0$, such that

$$
T^{\prime}\left[\begin{array}{cc}
P A+A^{\prime} P & P B-C^{\prime} \\
B^{\prime} P-C & 4 \sigma I_{m}-D-D^{\prime}
\end{array}\right] T \leqslant 0 ;
$$

and it is output-feedback passive with respect to $(\mathcal{U}, \mathcal{Y})$ with passivity margin $\sigma \in \mathbb{R}$ if there exists a matrix $P \in \mathbb{R}^{n \times n}$, $P=P^{\prime}>0$, such that

$$
T^{\prime}\left[\begin{array}{cc}
P A+A^{\prime} P+4 \sigma C^{\prime} C & P B-C^{\prime}+4 \sigma C^{\prime} D \\
B^{\prime} P-C+4 \sigma D^{\prime} C & 4 \sigma D^{\prime} D-D-D^{\prime}
\end{array}\right] T \leqslant 0,
$$

In all these cases, the storage function and the projection function can be chosen of the form

$$
S(x):=\frac{1}{2} x^{\prime} P x, \forall x \in \mathbb{R}^{n}, \quad \pi(u)=U u, \forall u \in \mathbb{R}^{m} .
$$

To prove Theorem 2 we need the following result showing that the commutation condition (6) will allow us to establish the existence of the solutions $(\bar{u}, \bar{x}, \bar{y})$ to (5) that appear in the inequalities (2a)-(2d), which are needed for the definition of passivity with respect to manifolds.

Lemma 1 (Commuting solutions). Suppose that the pair of projection matrices $U, Y \in \mathbb{R}^{m \times m}$ commutes with the LTI system (5) and consider the projection function $\pi$ defined in (8e). For every solution $(u, x, y)$ to (5) there is another solution $(\bar{u}, \bar{x}, \bar{y})$ to (5) with $\bar{u}(t)=\pi(u(t))$ and $\bar{y}(t) \in \operatorname{Im} Y$, $\forall t \geqslant 0$. Moreover, for every such solution we have $\bar{y}(t)=$ $C e^{A t} \bar{x}(0)+Y\left(y(t)-C e^{A t} x(0)\right)$, with $C e^{A t} \bar{x}(0) \in \operatorname{Im} Y$, $\forall t \geqslant 0$.

Proof of Lemma 1. Any solution $(\bar{u}, \bar{x}, \bar{y})$ to (5) with $\bar{u}=U u$ is given by

$$
\bar{y}(t)=C e^{A t} \bar{x}(0)+C \int_{0}^{t} e^{A(t-\tau)} B U u(\tau) d \tau+D U u(t) .
$$

From (7b), it then follows that

$$
\begin{aligned}
\bar{y}(t) & =C e^{A t} \bar{x}(0)+Y C \int_{0}^{t} e^{A(t-\tau)} B u(\tau) d \tau+Y D u(t), \\
& =C e^{A t} \bar{x}(0)+Y\left(y(t)-C e^{A t} x(0)\right),
\end{aligned}
$$

which shows that a necessary and sufficient condition for $\bar{y}(t) \in \operatorname{Im} Y, \forall t \geqslant 0$ is that $C e^{A t} \bar{x}(0) \in \operatorname{Im} Y, \forall t \geqslant 0$ (e.g. this can be achieved by setting $\bar{x}(0)=0$ ).

Proof of Theorem 2. For an arbitrary solution $(u, x, y)$ to (5), let $(\bar{u}, \bar{x}, \bar{y})$ be any solution to (5) whose existence is guaranteed by Lemma 1. Defining $\tilde{x}:=x-\bar{x}, \tilde{u}:=u-\bar{u}=$ $\left(I_{m}-U\right) u, \tilde{y}:=y-\bar{y}$, we have that

$$
\dot{\tilde{x}}=A \tilde{x}+B \tilde{u}, \quad \tilde{y}=C \tilde{x}+D \tilde{u},
$$

and therefore, for $s(t):=S(\tilde{x}(t))=\frac{1}{2} \tilde{x}(t)^{\prime} P \tilde{x}(t), \forall t \geqslant 0$, and $T:=\left[\begin{array}{cc}I_{n} & 0 \\ 0 & I_{m}-U\end{array}\right]$, we have that

$$
\begin{aligned}
\dot{s}-\tilde{u}^{\prime} \tilde{y} & =\frac{1}{2} \tilde{x}^{\prime}\left(P A+A^{\prime} P\right) \tilde{x}+\tilde{x}^{\prime} P B \tilde{u}-\tilde{u}^{\prime}(C \tilde{x}+D \tilde{u}), \\
& =\frac{1}{2}\left[\begin{array}{ll}
\tilde{x}^{\prime} & \tilde{u}^{\prime}
\end{array}\right]\left[\begin{array}{cc}
P A+A^{\prime} P & P B-C^{\prime} \\
B^{\prime} P-C & -D-D^{\prime}
\end{array}\right]\left[\begin{array}{l}
\tilde{x} \\
\tilde{u}
\end{array}\right], \\
& =\frac{1}{2}\left[\begin{array}{ll}
\tilde{x}^{\prime} & u^{\prime}
\end{array}\right] T^{\prime}\left[\begin{array}{cc}
P A+A^{\prime} P & P B-C^{\prime} \\
B^{\prime} P-C & -D-D^{\prime}
\end{array}\right] T\left[\begin{array}{l}
\tilde{x} \\
u
\end{array}\right] .
\end{aligned}
$$

When (8a) holds, this leads to (2a). If instead (8b) holds, we conclude that

$$
\begin{aligned}
\dot{s}-\tilde{u}^{\prime} \tilde{y} & \leqslant \frac{1}{2}\left[\begin{array}{ll}
\tilde{x}^{\prime} & u^{\prime}
\end{array}\right] T^{\prime}\left[\begin{array}{cc}
-2 \sigma P & 0 \\
0 & 0
\end{array}\right] T\left[\begin{array}{l}
\tilde{x} \\
u
\end{array}\right] \\
& =-\sigma \tilde{x}^{\prime} P \tilde{x}=-2 \sigma s,
\end{aligned}
$$

which leads to (2d); when (8c) holds, we conclude that

$$
\begin{aligned}
\dot{s}-\tilde{u}^{\prime} \tilde{y} & \leqslant \frac{1}{2}\left[\begin{array}{ll}
\tilde{x}^{\prime} & u^{\prime}
\end{array}\right] T^{\prime}\left[\begin{array}{cc}
0 & 0 \\
0 & -4 \sigma I_{m}
\end{array}\right] T\left[\begin{array}{l}
\tilde{x} \\
u
\end{array}\right] \\
& =-2 \sigma u^{\prime}\left(I_{m}-U\right)^{\prime}\left(I_{m}-U\right) u=-2 \sigma\|\tilde{u}\|^{2},
\end{aligned}
$$

which leads to (2b); and when (8d) holds, we conclude that

$$
\begin{aligned}
\dot{s}-\tilde{u}^{\prime} \tilde{y} & \leqslant \frac{1}{2}\left[\begin{array}{ll}
\tilde{x}^{\prime} & u^{\prime}
\end{array}\right] T^{\prime}\left[\begin{array}{ll}
-4 \sigma C^{\prime} C & -4 \sigma C^{\prime} D \\
-4 \sigma D^{\prime} C & -4 \sigma D^{\prime} D
\end{array}\right] T\left[\begin{array}{l}
\tilde{x} \\
u
\end{array}\right] \\
& =-2 \sigma\|\tilde{y}\|^{2},
\end{aligned}
$$

which leads to $(2 \mathrm{c})$. 


\section{Establishing passivity by composition}

A key feature of passivity-like properties is that one can establish the passivity of large systems by showing that these systems can be constructed by appropriate compositions of smaller passive subsystems. We present here two results of this nature that will be used in proving the main synchronization result in Section III-A: one for feedback interconnections, and another for diagonal compositions of equal linear systems ${ }^{2}$.

a) Feedback interconnection.: Consider two systems $\Sigma_{1}$ and $\Sigma_{2}$ of the form

$$
\Sigma_{k}: \quad \dot{x}_{k}=f_{k}\left(x_{k}, u_{k}\right), \quad y_{k}=g_{k}\left(x_{k}, u_{k}\right), \quad k \in\{1,2\},
$$

with $x_{k} \in \mathbb{R}^{n_{k}}, u_{k}, y_{k} \in \mathbb{R}^{m}$, and their negative feedback interconnection $\Sigma_{\text {feedback }}$ with input $u \in \mathbb{R}^{m}$ and output $y \in$ $\mathbb{R}^{m}$ defined by

$$
u_{1}(t)=u(t)-y_{2}(t), \quad u_{2}(t)=y_{1}(t)=: y(t), \quad \forall t \geqslant 0 .
$$

Lemma 2 (Feedback). Let $\mathcal{U}$ and $\mathcal{Y}$ be linear subspaces of $\mathbb{R}^{m}$ and $\pi_{u}$ and $\pi_{y}$ linear projections of $\mathbb{R}^{m}$ onto $\mathcal{U}$ and $\mathcal{Y}$, respectively.

C4 If $\Sigma_{1}$ is strictly-passive with respect to $(\mathcal{U}, \mathcal{Y})$ with projection function $\pi_{u}$ and passivity margin $\sigma_{\mathrm{s}, 1}>0$, and $\Sigma_{2}$ is strictly-passive with respect to ${ }^{3}(\mathcal{Y}, \mathcal{U})$ with projection function $\pi_{y}$ and passivity margin $\sigma_{\mathrm{s}, 2}>0$, then $\Sigma_{\text {feedback }}$ is passive with respect to $(\mathcal{U}, \mathcal{Y})$ with projection function $\pi_{u}$ and passivity margin $\min \left\{\sigma_{\mathrm{s}, 1}, \sigma_{\mathrm{s}, 2}\right\}>$ 0 .

C5 If $\Sigma_{1}$ is output-feedback passive with respect to $(\mathcal{U}, \mathcal{Y})$ with projection function $\pi_{u}$ and passivity margin $\sigma_{\mathrm{o}, 1} \in$ $\mathbb{R}$ and $\Sigma_{2}$ is input-feedthrough passive with respect to $(\mathcal{Y}, \mathcal{U})$ with projection function $\pi_{y}$ and margin $\sigma_{\mathrm{i}, 2} \in \mathbb{R}$, then $\Sigma_{\text {feedback }}$ is output-feedback passive with respect to $(\mathcal{U}, \mathcal{Y})$ with projection function $\pi_{u}$ and passivity margin $\sigma_{\mathrm{o}, 1}+\sigma_{\mathrm{i}, 2} \in \mathbb{R}$.

C6 If $\Sigma_{1}$ is input-feedthrough passive with respect to $(\mathcal{U}, \mathcal{Y})$ with projection function $\pi_{u}$ and passivity margin $\sigma_{\mathrm{i}, 1} \in$ $\mathbb{R}$ and $\Sigma_{2}$ is output-feedback passive with respect to $(\mathcal{Y}, \mathcal{U})$ with projection function $\pi_{y}$ and margin $\sigma_{\mathrm{o}, 2} \in \mathbb{R}$, then $\Sigma_{\text {feedback }}$ is input-strictly passive with respect to $(\mathcal{U}, \mathcal{Y})$ with projection function $\pi_{u}$ and passivity margin

$$
\sigma= \begin{cases}\frac{\sigma_{\mathrm{i}, 1} \sigma_{\mathrm{o}, 2}}{\sigma_{\mathrm{i}, 1}+\sigma_{\mathrm{o}, 2}}, & \text { if } \sigma_{\mathrm{i}, 1}>0, \sigma_{\mathrm{o}, 2}>0 \\ \frac{\sigma_{\mathrm{i}, 1}}{2}, & \text { if } \sigma_{\mathrm{i}, 1} \geqslant 0,-2 \sigma_{\mathrm{i}, 1} \leqslant \sigma_{\mathrm{o}, 2} \leqslant 0 \\ \frac{\sigma_{2}}{2} & \text { if } \sigma_{\mathrm{o}, 2} \geqslant 0,-2 \sigma_{\mathrm{o}, 2} \leqslant \sigma_{\mathrm{i}, 1} \leqslant 0\end{cases}
$$

for any of these three cases.

Proof of Lemma 2. For an arbitrary solution $(u, x, y)$ to $\Sigma_{\text {feedback, let }}$ us denote by $\left(u_{1}, x_{1}, y_{1}\right)$ and $\left(u_{2}, x_{2}, y_{2}\right)$ the corresponding solutions to the subsystems $\Sigma_{1}$ and $\Sigma_{2}$, respectively. From the passivity assumptions of $\Sigma_{1}$ and $\Sigma_{2}$,

\footnotetext{
${ }^{2}$ It is worth mentioning that one could prove similar results also for parallel interconnections of linear systems.

${ }^{3}$ We draw the reader's attention to the reverse order of the manifolds and projections.
}

we conclude that there exist other solutions $\left(\bar{u}_{1}, \bar{x}_{1}, \bar{y}_{1}\right)$ and $\left(\bar{u}_{2}, \bar{x}_{2}, \bar{y}_{2}\right)$ to the subsystems for which

$$
\begin{aligned}
& \bar{u}_{1}=\pi_{u}\left(u_{1}\right), \quad \bar{y}_{1} \in \mathcal{Y}, \\
& \dot{s}_{1} \leqslant\left(\tilde{y}_{1}\right)^{\prime}\left(\tilde{u}_{1}\right)-2 \sigma_{\mathrm{i}, 1}\left\|\tilde{u}_{1}\right\|-2 \sigma_{\mathrm{o}, 1}\left\|\tilde{y}_{1}\right\|-2 \sigma_{\mathrm{s}, 1} s_{1} ; \\
& \bar{u}_{2}=\pi_{y}\left(u_{2}\right), \quad \bar{y}_{2} \in \mathcal{U}, \\
& \dot{s}_{2} \leqslant\left(\tilde{y}_{2}\right)^{\prime}\left(\tilde{u}_{2}\right)-2 \sigma_{\mathrm{i}, 2}\left\|\tilde{u}_{2}\right\|-2 \sigma_{\mathrm{o}, 2}\left\|\tilde{y}_{2}\right\|-2 \sigma_{\mathrm{s}, 2} s_{2} ;
\end{aligned}
$$

where $s_{k}(t):=S_{k}\left(x_{k}(t)-\bar{x}_{k}(t)\right)$ and $S_{k}$ denotes the storage function of the system $\Sigma_{k}$. The constants $\sigma_{\mathrm{i}, \mathrm{k}}, \sigma_{\mathrm{o}, \mathrm{k}}, \sigma_{\mathrm{s}, \mathrm{k}}, k \in$ $\{1,2\}$ may be zero or not depending on the type of passivity of $\Sigma_{1}$ and $\Sigma_{2}$. Since $u_{1}=u-y_{2} \quad \Rightarrow \quad \bar{u}_{1}=\pi_{u}\left(u_{1}\right)=\pi_{u}(u-$ $\left.y_{2}\right)=\pi_{u}(u)-\pi_{u}\left(y_{2}\right)=\pi_{u}(u)-\bar{y}_{2}$, and $u_{2}=y_{1}=y \quad \Rightarrow$ $\bar{u}_{2}=\pi_{y}\left(u_{2}\right)=\pi_{y}\left(y_{1}\right)=\bar{y}_{1}=\bar{y} \in \mathcal{Y}$, we conclude that the solutions $\left(\bar{u}_{1}, \bar{x}_{1}, \bar{y}_{1}\right)$ and $\left(\bar{u}_{2}, \bar{x}_{2}, \bar{y}_{2}\right)$ satisfy (9) for an input equal to $\pi_{u}(u)$, leading to the output $\bar{y} \in \mathcal{Y}$. We can therefore use these solutions to construct a solution $(\bar{u}, \bar{x}, \bar{y})$ to $\Sigma_{\text {feedback }}$ with $\bar{u}:=\pi_{u}(u)$ (and, incidentally, $\bar{y}:=\pi_{y}(y)$ ). If we now consider the storage function $S\left(x_{1}, x_{2}\right):=S_{1}\left(x_{1}\right)+S_{2}\left(x_{2}\right)$, such that $\dot{s}=\dot{s}_{1}+\dot{s}_{2}$, we conclude from (10) that

$$
\begin{aligned}
\dot{s} \leqslant & -2 \sigma_{\mathrm{i}, 1}\left\|\tilde{u}_{1}\right\|^{2}-2 \sigma_{\mathrm{o}, 1}\left\|\tilde{y}_{1}\right\|^{2}-2 \sigma_{\mathrm{s}, 1} s_{1} \\
& -2 \sigma_{\mathrm{i}, 2}\left\|\tilde{u}_{2}\right\|^{2}-2 \sigma_{\mathrm{o}, 2}\left\|\tilde{y}_{2}\right\|^{2}-2 \sigma_{\mathrm{s}, 2} s_{2} \\
& +\left(\tilde{y}_{1}\right)^{\prime}\left(\tilde{u}_{1}\right)+\left(\tilde{y}_{2}\right)^{\prime}\left(\tilde{u}_{2}\right), \\
\leqslant & -2 \sigma_{\mathrm{i}, 1}\left\|u-y_{2}-\bar{u}+\bar{y}_{2}\right\|^{2}-2 \sigma_{\mathrm{o}, 1}\|\tilde{y}\|^{2}-2 \sigma_{\mathrm{s}, 1} s_{1} \\
& -2 \sigma_{\mathrm{i}, 2}\|\tilde{y}\|^{2}-2 \sigma_{\mathrm{o}, 2}\left\|\tilde{y}_{2}\right\|^{2}-2 \sigma_{\mathrm{s}, 2} s_{2} \\
& +(\tilde{y})^{\prime}\left(u-y_{2}-\bar{u}+\bar{y}_{2}\right)+\left(\tilde{y}_{2}\right)^{\prime}(\tilde{y}), \\
\leqslant & -2\left(\sigma_{\mathrm{o}, 1}+\sigma_{\mathrm{i}, 2}\right)\|\tilde{y}\|^{2}-2 \min \left\{\sigma_{\mathrm{s}, 1}, \sigma_{\mathrm{s}, 2}\right\}\left(s_{1}+s_{2}\right) \\
& -2 \sigma_{\mathrm{i}, 1}\left\|u-y_{2}-\bar{u}+\bar{y}_{2}\right\|^{2}-2 \sigma_{\mathrm{o}, 2}\left\|\tilde{y}_{2}\right\|^{2} \\
& +(\tilde{y})^{\prime}(\tilde{u}),
\end{aligned}
$$

where $s(t):=S\left(x_{1}(t)-\bar{x}_{1}(t), x_{2}(t)-\bar{x}_{2}(t)\right)$. The statements $\mathrm{C} 4$ and $\mathrm{C} 5$ follow directly from the inequality above. For the remaining cases, we need to construct appropriate bounds for the term $-2 \sigma_{\mathrm{i}, 1}\left\|u-y_{2}-\bar{u}+\bar{y}_{2}\right\|^{2}-2 \sigma_{\mathrm{o}, 2}\left\|y_{2}-\bar{y}_{2}\right\|^{2}$. In case $\sigma_{\mathrm{i}, 1}, \sigma_{\mathrm{o}, 2}>0$, we can use Proposition 2 in the appendix to conclude that

$$
-2 \sigma_{\mathrm{i}, 1}\left\|\tilde{u}-\tilde{y}_{2}\right\|^{2}-2 \sigma_{\mathrm{o}, 2}\left\|\tilde{y}_{2}\right\|^{2} \leqslant-\frac{2 \sigma_{\mathrm{i}, 1} \sigma_{\mathrm{o}, 2}}{\sigma_{\mathrm{i}, 1}+\sigma_{\mathrm{o}, 2}}\|\tilde{u}\|^{2} .
$$

In case $\sigma_{\mathrm{i}, 1} \leqslant 0$ and $\sigma_{\mathrm{i}, 1}+2 \sigma_{\mathrm{o}, 2} \geqslant 0$ we can also use Proposition 2 to conclude that

$$
\begin{aligned}
& -2 \sigma_{\mathrm{i}, 1}\left\|\tilde{u}-\tilde{y}_{2}\right\|^{2}-2 \sigma_{\mathrm{o}, 2}\left\|\tilde{y}_{2}\right\|^{2} \leqslant \\
& \quad-\sigma_{\mathrm{i}, 1}\|\tilde{u}\|^{2}-\sigma_{\mathrm{i}, 1}\left\|\tilde{y}_{2}\right\|^{2}-2 \sigma_{\mathrm{o}, 2}\left\|\tilde{y}_{2}\right\|^{2} \leqslant-\sigma_{\mathrm{i}, 1}\|\tilde{u}\|^{2} .
\end{aligned}
$$

In case $\sigma_{\mathrm{o}, 2} \leqslant 0$ and $2 \sigma_{\mathrm{i}, 1}+\sigma_{\mathrm{o}, 2} \geqslant 0$ we can also use Proposition 2 to conclude that

$$
\begin{aligned}
& -2 \sigma_{\mathrm{i}, 1}\left\|\tilde{u}-\tilde{y}_{2}\right\|^{2}-2 \sigma_{\mathrm{o}, 2}\left\|\tilde{y}_{2}\right\|^{2} \leqslant \\
& -2 \sigma_{\mathrm{i}, 1}\left\|\tilde{u}-\tilde{y}_{2}\right\|^{2}-\sigma_{\mathrm{o}, 2}\left\|\tilde{u}-\tilde{y}_{2}\right\|^{2}-\sigma_{\mathrm{o}, 2}\|\tilde{u}\|^{2} \leqslant-\sigma_{\mathrm{o}, 2}\|\tilde{u}\|^{2} .
\end{aligned}
$$

The following result regarding diagonal interconnections of linear systems is presented without proof, since it can be easily derived ${ }^{4}$.

\footnotetext{
${ }^{4}$ These proofs can be provided by the authors upon request.
} 
b) Diagonal composition.: Consider a system $\Sigma_{\text {diag }}$ composed of $N$ decoupled linear subsystems with equal dynamics:

$$
\Sigma_{k}: \quad \dot{x}_{k}=A x_{k}+B u_{k}, \quad y_{k}=C x_{k}+D u_{k},
$$

with $x_{k} \in \mathbb{R}^{n_{k}}, u_{k}, y_{k} \in \mathbb{R}^{m}, \forall k \in\{1,2, \ldots, N\}$. The overall system $\Sigma_{\text {diag }}$ has input $u:=\left(u_{1}, u_{2}, \ldots, u_{N}\right) \in \mathbb{R}^{N m}$ and output $y:=\left(y_{1}, y_{2}, \ldots, y_{N}\right) \in \mathbb{R}^{N m}$.

Lemma 3 (Diagonal composition). Consider a pair of linear subspaces $\mathcal{U}$ and $\mathcal{Y}$ of $\mathbb{R}^{m}$ and a projection function $\pi$ of $\mathbb{R}^{N m}$ onto $\underbrace{\mathcal{U} \times \mathcal{U} \ldots \times \mathcal{U}}_{N \text { times }} \equiv \mathcal{U}^{N}$ of the form

$$
\pi\left(u_{1}, u_{2}, \ldots, u_{N}\right)=\left(\sum_{i=1}^{N} \alpha_{i}^{1} u_{i}, \sum_{i=1}^{N} \alpha_{i}^{2} u_{i} \ldots, \sum_{i=1}^{N} \alpha_{i}^{N} u_{i}\right),
$$

for scalars $\alpha_{i}^{k} \in \mathbb{R}, \forall i, k \in\{1,2, \ldots, N\}$. In this case, the corresponding output will be given by

$$
\bar{y}=\left(\bar{y}_{1}, \bar{y}_{2}, \ldots, \bar{y}_{N}\right)=\left(\sum_{i=1}^{N} \alpha_{i}^{1} y_{i}, \sum_{i=1}^{N} \alpha_{i}^{2} y_{i} \ldots, \sum_{i=1}^{N} \alpha_{i}^{N} y_{i}\right) .
$$

C7 If all subsystems $\Sigma_{k}$ are strictly passive with respect to $(\{0\},\{0\})$ with passivity margin $\sigma>0$ then $\Sigma_{\text {diag }}$ is strictly passive with respect to $\left(\mathcal{U}^{N}, \mathcal{Y}^{N}\right)$ with a projection function $\pi$ and passivity margin $\sigma>0$.

C8 If all subsystems $\Sigma_{k}$ are input-feedforward passive with respect to $(\{0\},\{0\})$ with passivity margin $\sigma \in \mathbb{R}$ then $\Sigma_{\text {diag }}$ is input-feedforward passive with respect to $\left(\mathcal{U}^{N}, \mathcal{Y}^{N}\right)$ with a projection function $\pi$ and passivity margin $\sigma \in \mathbb{R}$.

C9 If all subsystems $\Sigma_{k}$ are output-feedback passive with respect to $(\{0\},\{0\})$ with passivity margin $\sigma \in \mathbb{R}$ then $\Sigma_{\text {diag }}$ is output-feedback passive with respect to $\left(\mathcal{U}^{N}, \mathcal{Y}^{N}\right)$ with a projection function $\pi$ and passivity margin $\sigma \in \mathbb{R}$.

\section{SYNCHRONIZATION IN ELECTRICAL NETWORKS}

We now focus our attention on the problem of synchronizing a group of $N$ identical oscillatory systems - henceforth called agents - whose dynamics are coupled through an interconnection network that is also a dynamical system. We view this problem from a design perspective, in the sense that we are given an interconnection network and our goal is to design the dynamics of the agents to make sure that the overall system synchronizes regardless of the initial conditions for the agents and the initial conditions for the electrical network that interconnects them.

a) Oscillator dynamics.: Following the work in $[11,26]$ we consider agents that are feedback oscillators consisting of an asymptotically stable LTI system in positive feedback with a nonlinear function $\phi: \mathbb{R} \rightarrow \mathbb{R}$. This class of oscillatory systems includes various well-known oscillators such as van der Pol oscillator [27], and the FitzHugh-Nagumo system [28]. Specifically, each agent $\mathrm{A}_{k}, \forall k \in\{1,2, \ldots, N\}$ is of the form

$$
\begin{aligned}
& \dot{x}_{k}=A_{\mathrm{a}} x_{k}+B_{\mathrm{a}} \hat{u}_{k}, \quad y_{k}^{\mathrm{a}}=C_{\mathrm{a}} x_{k}+D_{\mathrm{a}} \hat{u}_{k}, \\
& \hat{u}_{k}=u_{k}^{\mathrm{a}}+\phi\left(y_{k}^{\mathrm{a}}\right),
\end{aligned}
$$

with state vector $x_{k} \in \mathbb{R}^{n}$, scalar input $u_{k}^{\mathrm{a}} \in \mathbb{R}$, and scalar output $y_{k}^{\text {a }}$ (see Figure 3). All $N$ agents are assumed to share the

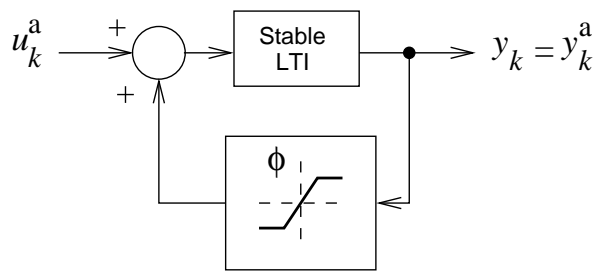

Figure 3. Block diagram for the dynamics of agent $A_{k}, k \in\{1,2, \ldots, N\}$.

same dynamics and our goal is for the agents to asymptotically synchronize in the sense that we want $y_{i}^{\mathrm{a}}(t)-y_{j}^{\mathrm{a}}(t) \rightarrow 0$ as $t \rightarrow \infty, \forall i, j \in\{1,2, \ldots, \mathbb{N}\}$. In addition, since we desire persistent oscillations, synchronization should not occur through convergence to an equilibrium point. Therefore the overall closed-loop system should not have any stable equilibrium point, but the solutions should remain bounded. These objectives will naturally impose additional constraints on the choice of the nonlinear function $\phi(\cdot)$ in (11), as it will become clear in sections III-A and III-C.

Assumption 1 (Agents). The following is assumed about the agents' dynamics (11):

\section{A1 The matrix $A_{\mathrm{a}}$ is Hurwitz.}

b) Interconnection network: The agents' dynamics are coupled through an LTI system of the form

$$
\dot{x}_{\mathrm{n}}=A_{\mathrm{n}} x_{\mathrm{n}}+B_{\mathrm{n}} u_{\mathrm{n}}, \quad y_{\mathrm{n}}=C_{\mathrm{n}} x_{\mathrm{n}}+D_{\mathrm{n}} u_{\mathrm{n}},
$$

with state vector $x_{\mathrm{n}} \in \mathbb{R}^{m}$, input vector $u_{\mathrm{n}} \in \mathbb{R}^{N}$, and output vector $y_{\mathrm{n}} \in \mathbb{R}^{N}$. The connections between agents through the network (12) corresponds to

$$
u_{\mathrm{a}}=-y_{\mathrm{n}}, \quad y_{\mathrm{a}}=u_{\mathrm{n}} .
$$

where $u_{\mathrm{a}}:=\left[\begin{array}{llll}u_{1}^{\mathrm{a}} & u_{2}^{\mathrm{a}} & \cdots & u_{N}^{\mathrm{a}}\end{array}\right]^{\prime} \in \mathbb{R}^{N}$, and $y_{\mathrm{a}}:=$ $\left[\begin{array}{llll}y_{1}^{\mathrm{a}} & y_{2}^{\mathrm{a}} & \cdots & y_{N}^{\mathrm{a}}\end{array}\right]^{\prime} \in \mathbb{R}^{N}$. Since we are considering equal agents, and we are interested in defining their consensus/synchronization as equality of their outputs, by assuming that each agent determines a unique and well-defined map between its input and output signals, we must consider interconnection networks that are symmetric from the point of view of their access ports, otherwise the problem would not be well-posed since each agent would have to exhibit the same output for different input signals, after achieving the synchronization condition. The interconnection networks we are interested in arise from electrical networks composed of inductors, resistors, capacitors, and multi-linear transformers. Notice that, interestingly, this arrangement (equal agents and symmetric network) naturally leads to equal sharing of energy among the agents.

When the vectors $u_{\mathrm{n}}$ and $y_{\mathrm{n}}$ in (12) are associated, respectively, with voltages and currents at the electrical network ports, symmetric networks are equivalent to star-shaped electrical circuits like the one depicted in Figure 2, with equal connecting admittances $g_{1}(s)=g_{2}(s)=\ldots=g_{N}(s)=g_{\mathrm{b}}(s)$, a 
load admittance $g_{\mathrm{L}}(s)$, and a transfer matrix of the form:

$$
G(s)=G^{\prime}(s)=g_{\mathrm{b}}(s) I_{N}-\frac{g_{\mathrm{b}}(s)^{2}}{N g_{\mathrm{b}}(s)+g_{\mathrm{L}}(s)} \overrightarrow{1} \overrightarrow{1}^{\prime},
$$

where $\overrightarrow{1} \in \mathbb{R}^{N}$ denotes a column vector with all entries equal to one. The scalar transfer matrices $g_{\mathrm{b}}(s)$ and $g_{\mathrm{L}}(s)$ are positivereal rational functions, since they represent admittances corresponding to associations of passive components [17].

Assumption 2 (Network). The following is assumed about the interconnection network (12):

A2 The matrix $A_{\mathrm{n}}$ is Hurwitz.

A3 The transfer function

$$
G(s)=C_{\mathrm{n}}\left(s I_{N}-A_{\mathrm{n}}\right)^{-1} B_{\mathrm{n}}+D_{\mathrm{n}}
$$

of (12) is of the form (14), where $g_{\mathrm{b}}(s)$ and $g_{\mathrm{L}}(s)$ are positive-real rational functions.

A4 The pair of matrices $(U, U)$, with $U:=\frac{1}{N} \overrightarrow{1} \overrightarrow{1}^{\prime}$, commutes with the system (12), i.e.

$$
G(s) U=U G(s), \quad \forall s \in \mathbb{C} .
$$

A5 The feedback interconnection of agents and network is well posed with locally Lipschitz continuous dynamics ${ }^{5}$.

The following result (proved in the appendix) states additional properties of $G(s)$ that follow from the preceding assumptions.

Lemma 4. Under Assumption 2, the eigenvalues of the transfer function $G(s)$, for each $s \in \mathbb{C}$, in (14) are $\lambda_{1}(s)=\lambda_{\mathrm{L}}(s)$, and $\lambda_{2}(s)=\lambda_{3}(s)=\cdots=\lambda_{N}(s)=\lambda_{\mathrm{b}}(s)$, with

$$
\begin{aligned}
& \lambda_{\mathrm{L}}(s)=\frac{g_{\mathrm{b}}(s) g_{\mathrm{L}}(s)}{N g_{\mathrm{b}}(s)+g_{\mathrm{L}}(s)}=\left(\frac{1}{\frac{g_{\mathrm{L}}(s)}{N}}+\frac{1}{g_{\mathrm{b}}(s)}\right)^{-1}, \\
& \lambda_{\mathrm{b}}(s)=g_{\mathrm{b}}(s)
\end{aligned}
$$

positive real rational functions. Moreover, the constant vector $\overrightarrow{1}$ is an eigenvector associated with the eigenvalue $\lambda_{\mathrm{L}}(s)$, i.e.,

$$
G(s) \overrightarrow{1}=\lambda_{\mathrm{L}}(s) \overrightarrow{1} \text {. }
$$

Remark 5 (Coupling under synchronization). Even when the agents are perfectly synchronized, i.e., $y_{1}^{\mathrm{a}}(t)=y_{2}^{\mathrm{a}}(t)=\ldots=$ $y_{N}^{\mathrm{a}}(t)$, the output of the interconnection network typically does not converge to zero and the agents' dynamics continue to be coupled. To verify that this is so, note that it follows from (19) that when the inputs to $G(s)$ are identical periodic signals, the forced response can only be identically zero if $\lambda_{\mathrm{L}}(s)$ has zeros over the imaginary axis and all the energy of the inputs is concentrated at those frequencies. In general, $\lambda_{\mathrm{L}}(s)$ has no such zeros. The existence of a coupling between agents, even under perfect synchronization, similarly to what was considered in [6] in the context of transient stability analysis of multimachine power networks, makes the present

\footnotetext{
${ }^{5}$ When the agents do not have direct feedthrough (i.e., $D_{\mathrm{a}}=0$ ) and $\phi$ is a locally Lipschitz continuous function, this holds for every interconnection network.
}

synchronization problem distinct from many problems reported in the literature on nonlinear dynamical systems synchronization and multi-agent consensus (e.g. see [19] and references therein).

\section{A. Synchronization Result}

The interconnection of the network (12) with the agents defined by (11) can be represented by the block diagram in Figure 4, where $G(s)$ denotes the network transfer function in (14), $A(s)$ denotes the transfer function of the diagonal composition of the LTI systems in (11a)

$$
A(s)=a(s) I_{N}, \quad a(s):=C_{\mathrm{a}}\left(s I_{n}-A_{\mathrm{a}}\right)^{-1} B_{\mathrm{a}}+D_{\mathrm{a}},
$$

and

$$
\Phi\left(y_{\mathrm{a}}\right):=\left[\phi\left(y_{1}^{\mathrm{a}}\right) \phi\left(y_{2}^{\mathrm{a}}\right) \ldots \phi\left(y_{N}^{\mathrm{a}}\right)\right]^{\prime}, \quad \forall y_{\mathrm{a}} \in \mathbb{R}^{N} .
$$

From (15) and (20), the feedback LTI system shown in the dashed box in Figure 4 corresponds to

$$
F(s)=\left[I_{N}+A(s) G(s)\right]^{-1} A(s) .
$$

A characteristic of this inner feedback LTI system (proved in the appendix) that will be important in the following development is the fact that it commutes with the pair of matrices $U, U \in \mathbb{R}^{N \times N}$.

Lemma 5. Under the assumption that a pair of matrices $U, U \in \mathbb{R}^{N \times N}$ commutes with $G(s)$, i.e. $U G(s)=G(s) U$, the same pair of matrices $U, U \in \mathbb{R}^{N \times N}$ also commutes with the system $F(s)$ in (21), i.e. $U F(s)=F(s) U$.

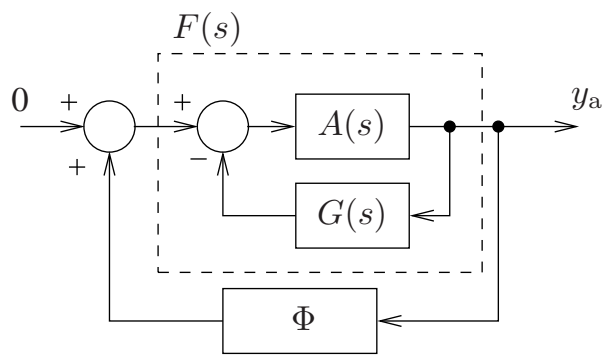

Figure 4. Block diagram representation of the overall system (11), (12), (13) consisting of the nonlinear agents connected through the electrical network.

The diagram in Figure 4 highlights that the system under study is of the general form considered in Theorem 1, which is the basis for the key result of this section concerning sufficient conditions under which the agents' outputs will become equal.

Theorem 3 (Synchronization). Suppose that Assumptions 1, 2 hold for the system (11) - (13). Any of the following two sets of conditions C10 or C11 suffices to guarantee that the agents asymptotically synchronize, in the sense that their inputs and/or outputs converge to the linear subspace $\operatorname{Im} U$, which corresponds to the equality of all the inputs and/or outputs.

C10 Suppose that

1) there exists a matrix $P_{\mathrm{n}} \in \mathbb{R}^{m \times m}, P_{\mathrm{n}}=P_{\mathrm{n}}^{\prime}>0$, such that, with $T:=\left[\begin{array}{cc}I_{m} & 0 \\ 0 & I_{N}-U\end{array}\right]$ for $U:=\frac{1}{N} \overrightarrow{1} \overrightarrow{1}^{\prime}$, 
$\sigma=\sigma_{\mathrm{n}} \in \mathbb{R}, P=P_{\mathrm{n}}, A=A_{\mathrm{n}}, B=B_{\mathrm{n}}$, $C=C_{\mathrm{n}}$ and $D=D_{\mathrm{n}}$, inequality (8c) is satisfied, and, therefore, the interconnection network (12) is input-feedforward passive with respect to $(\operatorname{Im} U, \operatorname{Im} U)$, with passivity margin $\sigma_{\mathrm{n}} \in \mathbb{R}$;

2) there exists a matrix $P_{\mathrm{a}} \in \mathbb{R}^{n \times n}, P_{\mathrm{a}}=P_{\mathrm{a}}^{\prime}>0$, such that, with $T:=I_{n+1}, \sigma=\sigma_{\mathrm{a}} \in \mathbb{R}$, $P=P_{\mathrm{a}}, A=A_{\mathrm{a}}, B=B_{\mathrm{a}}, C=C_{\mathrm{a}}$ and $D=D_{\mathrm{a}}$, inequality (8d) is satisfied, and, therefore (11a) is output-feedback passive in the usual sense, i.e., it is output-feedback passive with respect to $(\{0\},\{0\})$, with passivity margin $\sigma_{\mathrm{a}} \in \mathbb{R} ;$ and

$3)$ the nonlinearity $\phi(\cdot)$ in $(11 \mathrm{~b})$ satisfies

$$
\left(s_{2}-s_{1}\right)\left(\phi\left(s_{2}\right)-\phi\left(s_{1}\right)\right) \leqslant 2 \alpha\left(s_{2}-s_{1}\right)^{2},
$$

$\forall s_{1}, s_{2} \in \mathbb{R}$, with $\alpha<\sigma_{\mathrm{a}}+\sigma_{\mathrm{n}}$. A saturation function $\phi(y)$ with slope $\alpha<\sigma_{\mathrm{a}}+\sigma_{\mathrm{n}}$ at the origin satisfies this requirement.

Then both $u_{\mathrm{a}}=-y_{\mathrm{n}}$ and $y_{\mathrm{a}}=u_{\mathrm{n}}$ converge to the linear subspace $\mathcal{Y}:=\mathcal{U}:=\operatorname{Im} U, U:=\frac{1}{N} \overrightarrow{1} \overrightarrow{1}^{\prime}$ as $t \rightarrow \infty$, along every solution to (11), (12), (13) that is globally defined and uniformly bounded.

\section{C11 Suppose that}

1) there exists a matrix $P_{\mathrm{n}} \in \mathbb{R}^{m \times m}, P_{\mathrm{n}}=P_{\mathrm{n}}^{\prime}>0$, such that, with $T:=\left[\begin{array}{cc}I_{m} & 0 \\ 0 & I_{N}-U\end{array}\right]$ for $U:=\frac{1}{N} \overrightarrow{1} \overrightarrow{1}^{\prime}$, $\sigma=\sigma_{\mathrm{n}}>0, P=P_{\mathrm{n}}, A=A_{\mathrm{n}}, B=B_{\mathrm{n}}$, $C=C_{\mathrm{n}}$ and $D=D_{\mathrm{n}}$, inequality (8d) is satisfied, and, therefore, the interconnection network (12) is output-strictly passive with respect to $(\operatorname{Im} U, \operatorname{Im} U)$, with passivity margin $\sigma_{\mathrm{n}}>0$;

2) there exists a matrix $P_{\mathrm{a}} \in \mathbb{R}^{n \times n}, P_{\mathrm{a}}=P_{\mathrm{a}}^{\prime}>0$, such that, with $T:=I_{n+1}, \sigma=\sigma_{\mathrm{a}} \geqslant-2 \sigma_{\mathrm{n}}$, $P=P_{\mathrm{a}}, A=A_{\mathrm{a}}, B=B_{\mathrm{a}}, C=C_{\mathrm{a}}$ and $D=D_{\mathrm{a}}$, inequality (8c) is satisfied, and, therefore, (11a) is input-feedforward passive in the usual sense, i.e., it is input-feedforward passive with respect to $(\{0\},\{0\})$, with passivity margin $\sigma_{\mathrm{a}} \geqslant-2 \sigma_{\mathrm{n}}$; and $3)$ the nonlinearity $\phi(\cdot)$ in (11b) satisfies

$$
\left(s_{2}-s_{1}\right)\left(\phi\left(s_{2}\right)-\phi\left(s_{1}\right)\right) \leqslant 2 \alpha\left(\phi\left(s_{2}\right)-\phi\left(s_{1}\right)\right)^{2},
$$

$$
\begin{aligned}
& \forall s_{1}, s_{2} \in \mathbb{R}, \text { with } \\
& \begin{cases}\alpha<\frac{\sigma_{\mathrm{a}} \sigma_{\mathrm{n}}}{\sigma_{\mathrm{a}}+\sigma_{\mathrm{n}}}, & \text { if } \quad \sigma_{\mathrm{a}}>0, \\
\alpha<\frac{\sigma_{\mathrm{n}}}{2}, & \text { if } \quad-2 \sigma_{\mathrm{n}} \leqslant \sigma_{\mathrm{a}} \leqslant 0 .\end{cases}
\end{aligned}
$$

Then $u_{\mathrm{a}}=-y_{\mathrm{n}}$ converges to the linear subspace $\mathcal{Y}:=$ $\mathcal{U}:=\operatorname{Im} U, U:=\frac{1}{N} \overrightarrow{1} \overrightarrow{1}^{\prime}$ as $t \rightarrow \infty$, along every solution to (11), (12), (13) that is globally defined and uniformly bounded.

Remark 6 (Input/output versus input-only convergence). The condition C11 only guarantees that the inputs of the agents synchronize. To make sure that their outputs also synchronize one could require the nonlinearities $\phi$ to be injective and continuous, which would hold, e.g., for a "smooth" saturation like the arc-tangent function. Alternatively, one could require the transfer function $F(s)$ depicted inside the dashed box in Figure 4 [corresponding to the feedback connection between the LTI agent subsystems (11a) and the interconnection network (12)] to be asymptotically stable. We shall find in Section III-C sufficient conditions for this to hold, which also guarantee that every solution is globally defined and uniformly bounded. In addition, in Section IV it will become clear that condition $\mathrm{C11}$ is crucial to investigate the important practical case of electrical networks with inductive elements in the interconnecting branches.

To prove Theorem 3 we need the following technical lemma that we will use to establish the inequalities in Theorem 1.

Lemma 6. Suppose that the pair of projection matrices $U, U \in$ $\mathbb{R}^{m \times m}$, with $U:=\frac{1}{N} \overrightarrow{1} \overrightarrow{1}^{\prime}$, commutes with the LTI system (5) and that (22) holds. For every pair of solutions $(u, x, y)$ and $(\bar{u}, \bar{x}, \bar{y})$ to $(5)$ with $\bar{u}(t)=\pi(u(t)):=U u(t)$ and $\bar{y}(t) \in \operatorname{Im} U$, $\forall t \geqslant 0$ the inequality (4c) holds. Similarly, if we replace (22) by (23a), then (4b) holds instead of (4c).

Proof of Lemma 6. In view of Lemma 1 (with $Y=U$ ), the solutions $(u, x, y)$ and $(\bar{u}, \bar{x}, \bar{y})$ must be related by

$$
\bar{y}(t)=C e^{A t} \bar{x}(0)+U\left(y(t)-C e^{A t} x(0)\right)=U y(t)+r(t),
$$

with $C e^{A t} \bar{x}(0) \in \operatorname{Im} U$ and $r(t):=C e^{A t} \bar{x}(0)-U C e^{A t} x(0) \in$ $\operatorname{Im} U, \forall t \geqslant 0$. We can therefore re-write the left-hand side of (4c) as

$$
(M \Phi(y))^{\prime}(y-\bar{y})=\Phi(y)^{\prime} M(M y-r)=\Phi(y)^{\prime} M^{2} y,
$$

where $M:=I-U$ and the last equality is a consequence of the fact that $r \in \operatorname{Im} U$. Using the additional fact that $M^{2}=M$, this simplifies to

$$
\Phi(y)^{\prime} M y=\sum_{i=1}^{N} \phi\left(y_{i}\right)\left(y_{i}-\hat{y}\right),
$$

where $\hat{y}=\frac{1}{N} \sum_{i=1}^{N} y_{i}$. Adding and subtracting $\phi(\hat{y})$ to $\phi\left(y_{i}\right)$ and using (22), we conclude that

$$
\begin{aligned}
& (M \Phi(y))^{\prime}(y-\bar{y})=\sum_{i=1}^{N}\left(\phi\left(y_{i}\right)-\phi(\hat{y})+\phi(\hat{y})\right)\left(y_{i}-\hat{y}\right) \\
& \leqslant 2 \alpha \sum_{i=1}^{N}\left(y_{i}-\hat{y}\right)^{2}+\phi(\hat{y}) \sum_{i=1}^{N}\left(y_{i}-\hat{y}\right)=2 \alpha\|M y\|^{2},
\end{aligned}
$$

where the last equality is a consequence of the fact that $\sum_{i=1}^{N}\left(y_{i}-\hat{y}\right)=0$. Since $r \in \operatorname{Im} U$, the vector $r$ is orthogonal to $M y$ and therefore

$$
\begin{aligned}
\|y-\bar{y}\|^{2}=\|y-U y-r\|^{2}=\| & (I-U) y\left\|^{2}+\right\| r \|^{2} \\
& \Rightarrow\|M y\|^{2} \leqslant\|y-\bar{y}\|^{2} .
\end{aligned}
$$

Inequality (4c) follows from this and (25).

To prove (4b), we go back to (24) and re-write it as

$$
\begin{aligned}
&(M \Phi(y))^{\prime}(y-\bar{y})=(M \Phi(y))^{\prime} M y \\
&=(M \Phi(y))^{\prime}(M y-2 \alpha M \Phi(y))+2 \alpha\|M \Phi(y)\|^{2} \\
&=\sum_{i=1}^{N}\left(\phi\left(y_{i}\right)-\hat{\phi}(y)\right)^{\prime}\left(y_{i}-\hat{y}-2 \alpha \phi\left(y_{i}\right)+2 \alpha \hat{\phi}(y)\right) \\
&+2 \alpha\|M \Phi(y)\|^{2}
\end{aligned}
$$


where $\hat{\phi}(y):=\frac{1}{N} \sum_{i=1}^{N} \phi\left(y_{i}\right)$. Adding and subtracting $\phi(\hat{y})$ and using (23a), we conclude that

$$
\begin{aligned}
& (M \Phi(y))^{\prime}(y-\bar{y})-2 \alpha\|M \Phi(y)\|^{2} \\
= & \sum_{i=1}^{N}\left(\phi\left(y_{i}\right)-\phi(\hat{y})+\phi(\hat{y})-\hat{\phi}(y)\right)^{\prime}\left(y_{i}-\hat{y}-2 \alpha \phi\left(y_{i}\right)+2 \alpha \hat{\phi}(y)\right) \\
\leqslant & \sum_{i=1}^{N} 2 \alpha\left(\phi\left(y_{i}\right)-\phi(\hat{y})\right)^{2}-2 \alpha\left(\phi\left(y_{i}\right)-\phi(\hat{y})\right)\left(\phi\left(y_{i}\right)-\hat{\phi}(y)\right) \\
& \quad+(\phi(\hat{y})-\hat{\phi}(y))^{\prime}\left(y_{i}-\hat{y}-2 \alpha \phi\left(y_{i}\right)+2 \alpha \hat{\phi}(y)\right) .
\end{aligned}
$$

Since $\sum_{i=1}^{N} y_{i}-\hat{y}=\sum_{i=1}^{N} \phi\left(y_{i}\right)-\hat{\phi}(y)=0$ and also $\sum_{i=1}^{N}\left(\phi\left(y_{i}\right)-\phi(\hat{y})\right)^{2}-\left(\phi\left(y_{i}\right)-\phi(\hat{y})\right)\left(\phi\left(y_{i}\right)-\hat{\phi}(y)\right)=0$, we conclude that the above inequality simplifies to

$$
(M \Phi(y))^{\prime}(y-\bar{y}) \leqslant 2 \alpha\|M \Phi(y)\|^{2},
$$

which concludes the proof since this is precisely equivalent to (4b).

Proof of Theorem 3. We start by considering the conditions C10. From Theorem 2, since inequality (8d) is satisfied and the system (11a) commutes with the trivial pair of matrices $U=0, Y=0$, each LTI agent subsystem is output-feedback passive with respect to $(\{0\},\{0\})$, with passivity margin $\sigma_{\mathrm{a}} \in \mathbb{R}$. We then conclude from Lemma 3, condition C9, with $\alpha_{i}^{k}=\frac{1}{N}, \forall i, k \in\{1,2, \ldots, N\}$, and for $U=\frac{1}{N} \overrightarrow{1} \overrightarrow{1}^{\prime}$, that the diagonal composition of the LTI systems with dynamics (11a) is output-feedback passive with respect to $(\operatorname{Im} U, \operatorname{Im} U)$, for the projection function $\pi_{u}(z)=U z, \forall z \in \mathbb{R}^{N}$, with passivity margin $\sigma_{\mathrm{a}} \in \mathbb{R}$. Notice that (12) is input-feedforward passive with respect to $(\operatorname{Im} U, \operatorname{Im} U)$ for the projection function $\pi_{y}(z)=U z, \forall z \in \mathbb{R}^{N}$, with passivity margin $\sigma_{\mathrm{n}} \in \mathbb{R}$, because, from Assumption 2, (12) commutes with the pair $(U, U)$, and since the inequality (8c) is satisfied, we can apply Theorem 2. We can now use Lemma 2, condition C5, to conclude that the feedback connection between the LTI agent subsystems (11a) and the interconnection network (12) (which is depicted inside the dashed box in Figure 4) is output-feedback passive with respect to $(\operatorname{Im} U, \operatorname{Im} U)$, considering the projection function $\pi(z)=U z, \forall z \in \mathbb{R}^{N}$, with passivity margin $\sigma_{\mathrm{a}}+\sigma_{\mathrm{n}} \in \mathbb{R}$. To apply Theorem 1 to the feedback system shown in the dashed box in Figure 4, it remains to verify that (4c) holds with $\alpha<\sigma:=\sigma_{\mathrm{a}}+\sigma_{\mathrm{n}}$. From Lemma 5 it follows that $U F(s)=F(s) U$, which allow us to use Lemma 6 to conclude that the inequality (4c) indeed holds.

We now consider the conditions $\mathrm{C} 11$. From Theorem 2, since inequality (8c) is satisfied and the system (11a) commutes with the trivial pair of matrices $U=0, Y=0$, each LTI agent subsystem is input-feedforward passive with respect to $(\{0\},\{0\})$. We then conclude from Lemma 3, condition C8, with $\alpha_{i}^{k}=\frac{1}{N}, \forall i, k \in\{1,2, \ldots, N\}$, and for $U=$ $\frac{1}{N} \overrightarrow{1} \overrightarrow{1}^{\prime}$, that the diagonal composition of the LTI systems with dynamics (11a) is input-feedforward passive with respect to $(\operatorname{Im} U, \operatorname{Im} U)$ for the projection function $\pi_{u}(z)=U z$, $\forall z \in \mathbb{R}^{N}$, with passivity margin $\sigma_{\mathrm{a}} \in \mathbb{R}$. Since (12) commutes with the pair $(U, U)$ and $(8 \mathrm{~d})$ holds, we conclude from Theorem 2 that the interconnection network (12) is output-strictly passive with respect to $(\operatorname{Im} U, \operatorname{Im} U)$ for the projection function $\pi_{y}(z)=U z, \forall z \in \mathbb{R}^{N}$, with passivity margin $\sigma_{\mathrm{n}}>0$. We can now use Lemma 2, condition C6, to conclude that the feedback connection between the LTI agent subsystems (11a) and the interconnection network (12) (which is depicted inside a dashed box in Figure 4) is inputstrictly passive with respect to $(\operatorname{Im} U, \operatorname{Im} U)$, for the projection function $\pi(z)=U z, \forall z \in \mathbb{R}^{N}$, with passivity margin

$$
\sigma= \begin{cases}\frac{\sigma_{\mathrm{a}} \sigma_{\mathrm{n}}}{\sigma_{\mathrm{a}}+\sigma_{\mathrm{n}}}, & \text { if } \sigma_{\mathrm{a}}>0 \\ \frac{\sigma_{\mathrm{n}}}{2}, & \text { if }-2 \sigma_{\mathrm{n}} \leqslant \sigma_{\mathrm{a}} \leqslant 0 .\end{cases}
$$

To apply Theorem 1 to the feedback in Figure 4, it remains to verify that (4b) holds with $\sigma>\alpha$. From Lemma 5 it follows that $U F(s)=F(s) U$, which allow us to use Lemma 6 to conclude that the inequality (4b) indeed holds.

\section{B. Avoiding Unbounded Solutions or Trivial Synchronization}

Theorem 3 in the previous section provides conditions to guarantee that the inputs of the agents converge to each other, for every globally defined and uniformly bounded solution. However, in practice we would like to guarantee that synchronization does not occur by convergence to an equilibrium point, since the goal is to obtain synchronized oscillations. In addition, we would like to guarantee that every solution is globally defined and bounded.

From the block diagram of the overall system depicted in Figure 4 we conclude that if the nonlinearity $\Phi$ is bounded and the transfer function $F(s)$ is BIBO stable ${ }^{6}$, then every solution is globally defined and uniformly bounded solution.

Regarding the possibility of synchronization by convergence to an equilibrium point for which all agents exhibit the same constant output $y_{\text {eq }} \in \mathbb{R}$, one can also conclude from Figure 4 that the local (in)stability of such equilibrium point is determined by the (in)stability of the feedback connection between the transfer function $F(s)$ and the linearization of $\Phi$ around the equilibrium output, which is depicted in Figure 5. The following result summarizes these observations.

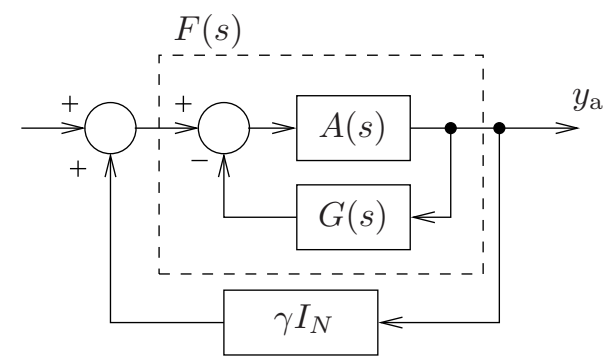

Figure 5. Block diagram representation of the local linearization of the overall system (11), (12), (13) around an equilibrium point for which all agents exhibit the same output $y_{\text {eq }} \in \mathbb{R}$, where $\gamma:=\frac{d \phi}{d y}\left(y_{\text {eq }}\right)$.

Proposition 1. Suppose that the agent's nonlinearity $\phi$ is bounded and that the LTI system represented by block diagram

\footnotetext{
${ }^{6}$ Since the blocks $A(s)$ and $G(s)$ in Figure 4 correspond to asymptotically stable linear systems (cf. Assumptions 1 and 2), one need not worry about unstable cancelations in their feedback interconnection.
} 
in Figure 5 is BIBO stable for $\gamma=0$ and unstable for $\gamma:=$ $\frac{d \phi}{d y}\left(y_{\mathrm{eq}}\right)$, with $y_{\mathrm{eq}} \in \mathbb{R}$ a constant value. Then every solution to the original system (11), (12), (13) is globally defined and bounded. Moreover, any equilibrium point corresponding to each agent having the same constant output value $y_{\mathrm{eq}} \in \mathbb{R}$ is unstable.

The instability of the linear system in Figure 5 depends on the non-trivial stability analysis of the resulting MultipleInput/Multiple-Output (MIMO) system. However, thanks to the symmetry of the problem (equal agents and symmetric interconnection network), this turns out to be easily performed through a root-locus analysis of only two Single-Input/SingleOutput (SISO) transfer functions, independently of the number of agents involved. This result was inspired by the bifurcation analysis proposed in [11], which was extended to account for a symmetric interconnection structure with memory.

Lemma 7. Suppose that Assumptions 1 and 2 hold. To determine the poles of the LTI system represented by the block diagram in Figure 5, in order to investigate the local linear stability of the system shown in Figure 4, is equivalent to find the roots $s \in \mathbb{C}$ of the following two characteristic equations:

$$
1-\gamma h_{\mathrm{L}}(s)=0, \quad 1-\gamma h_{\mathrm{b}}(s)=0,
$$

where $h_{\mathrm{L}}(s):=\frac{a(s)}{1+a(s) \lambda_{\mathrm{L}}(s)}$, and $h_{\mathrm{b}}(s):=\frac{a(s)}{1+a(s) \lambda_{\mathrm{b}}(s)}$, with $\lambda_{L}(s), \lambda_{b}(s)$, and $a(s)$ defined in (17), (18), and (20), respectively.

Notice that the analysis of the two root-loci mentioned above will be instrumental in Section III-C to design the nonlinearity $\phi$ in $(11 \mathrm{~b})$, in the sense that we will be able to choose the value of $\gamma:=\frac{d \phi}{d y}\left(y_{\text {eq }}\right)$ that avoids trivial synchronization by guaranteeing that the local linear dynamics around the equilibrium point is unstable.

Proof of Lemma 7. The transfer function of the LTI system represented by block diagram in Figure 5 is given by

$$
\begin{aligned}
T(s) & =\left(I_{N}-\gamma A(s)+A(s) G(s)\right)^{-1} A(s), \\
& =\left(\frac{1-\gamma a(s)}{a(s)} I_{N}+G(s)\right)^{-1},
\end{aligned}
$$

where we used the fact that $A(s)=a(s) I_{N}$. Therefore $s \in \mathbb{C}$ is a pole of the transfer function if and only if $\operatorname{det}\left(\frac{1-\gamma a(s)}{a(s)} I_{N}+\right.$ $G(s))=0$, which is to say that $-\frac{1-\gamma a(s)}{a(s)}$ is an eigenvalue of $G(s)$. In view of Lemma 4 , we conclude that $s \in \mathbb{C}$ is pole when either one of the following two conditions hold

$$
\begin{aligned}
& -\frac{1-\gamma a(s)}{a(s)}=\lambda_{L}(s) \quad \Leftrightarrow \quad 1-\gamma h_{L}(s)=0 \\
& -\frac{1-\gamma a(s)}{a(s)}=\lambda_{b}(s) \quad \Leftrightarrow \quad 1-\gamma h_{b}(s)=0,
\end{aligned}
$$

which concludes the proof.

\section{Constructive Approach to Synchronization}

Our goal in this section is to provide a step-by-step procedure to design each agent (11), particularly the nonlinear function $\phi$ in (11b), such that: $(i)$ there will be no unbounded solutions; and (ii) the agents will not reach synchronization by simply exhibiting the same constant value at their outputs:

Step 1. Determine any values $y_{\mathrm{eq}} \in \mathbb{R}$ for which the system could have an equilibrium point for which all agents exhibit the same output $y_{\text {eq }} \in \mathbb{R}$. In view of (19), such $y_{\text {eq }} \in \mathbb{R}$ must satisfy

$$
y_{\mathrm{eq}}=a(0) \phi\left(\left(1-\lambda_{L}(0)\right) y_{\mathrm{eq}}\right)
$$

where $\lambda_{L}(s)$ and $a(s)$ are defined in (17) and (20), respectively. Notice that $y_{\text {eq }}=0$ will always be a possible solution to this equation.

Step 2. Draw the two root-loci in (26) and determine the range $\Gamma_{\text {unstable }} \subset(0, \infty)$ of values of $\gamma$ for which at least one of the root loci has an unstable root. Note the minus sign in (26), which corresponds to a positive feedback and changes some of the rules normally used to sketch the root-locus.

Step 3. Verify that the LTI system $F(s)$ in Figure 5 is BIBO stable, such that every solution to (11), (12), (13) is globally defined and uniformly bounded solution. A possible conservative approach is to ensure that for $\gamma \downarrow 0^{+}$all roots of both root-loci - which are the roots of $1+a(s) \lambda_{\mathrm{L}}(s)$ and $1+a(s) \lambda_{\mathrm{b}}(s)$ - lie in the open left-hand side complex plane, since asymptotic stability implies BIBO stability in this case. Generically this will hold under the assumptions of Theorem 3 because this result requires the agents' transfer function $a(s)$ to be passive and both $\lambda_{\mathrm{L}}(s)$ and $\lambda_{\mathrm{b}}(s)$ are positive-real transfer functions (cf. Assumption 2) and, therefore, they are passive LTI systems too.

Step 4. Determine the largest value for $\sigma=\sigma_{\mathrm{n}}$ for which inequality (8c) is satisfied, with $T:=\left[\begin{array}{cc}I_{m} & 0 \\ 0 & I_{N}-U\end{array}\right], U:=\frac{1}{N} \overrightarrow{1} \overrightarrow{1^{\prime}}$, $P=P_{\mathrm{n}}, A=A_{\mathrm{n}}, B=B_{\mathrm{n}}, C=C_{\mathrm{n}}$ and $D=D_{\mathrm{n}}$. Determine also the largest value for $\sigma=\sigma_{\mathrm{a}}$ for which inequality (8d) holds, with $T:=I_{n+1}, P=P_{\mathrm{a}}, A=A_{\mathrm{a}}, B=B_{\mathrm{a}}, C=C_{\mathrm{a}}$ and $D=D_{\text {a }}$.

Step 5. Pick for $\phi$ a saturation function with slope at the origin equal to $\alpha \in \Gamma_{\text {unstable }} \cap\left(-\infty, \sigma_{\mathrm{n}}+\sigma_{\mathrm{a}}\right)$ and breakpoints sufficiently large so that $\frac{d \phi}{d y}\left(y_{\mathrm{eq}}\right)=\alpha$.

Remark 7 (Practical issues). In view of Lemma 7, Proposition 1, and Theorem 3, this guarantees that the agents will synchronize (since $\alpha<\sigma_{\mathrm{n}}+\sigma_{\mathrm{a}}$ ) and that this will not occur by convergence to a stable equilibrium point (since $\left.\frac{d \phi}{d y}\left(y_{\mathrm{eq}}\right) \in \Gamma_{\text {unstable }}\right)$. In practice, typically there will still exist equilibrium points embedded in stable manifolds. However, the local instability of the equilibria guarantees that these manifolds have dimensions that are strictly smaller than that of the state space and therefore the set of initial conditions from which the system will converge to these equilibria has Lebesgue measure zero. Among all possible values for $\alpha$ in $\Gamma_{\text {unstable }} \cap\left(-\infty, \sigma_{\mathrm{n}}+\sigma_{\mathrm{a}}\right)$, the largest value for $\alpha$ is typically desirable as it will lead to trajectories that most rapidly move away from the equilibrium point. The positions of the saturation breakpoints also determine the saturation levels, which can be used to enforce a desired amplitude for the oscillations [20]. 
The procedure outlined above may fail because the set $\Gamma_{\text {unstable }} \cap\left(-\infty, \sigma_{\mathrm{n}}+\sigma_{\mathrm{a}}\right)$ is empty. In this case, one would need to redesign the agent's transfer function $a(s)$ to make sure that one can get sustained synchronized oscillations. The usual rules used to sketch the root-locus can be useful to accomplish this task, with the caveat that we are now interested in making the system unstable instead of stable.

\section{Special case: electrical network with resistive intercon- necting branches}

In the context of small power networks, such as the ones relevant to the problem of designing redundant systems based on parallel connected Uninterruptible Power Supplies (UPS) (see, e.g., [2, 9, 29], and references therein), one could have essentially purely resistive connecting admittances if the cable lengths are short enough [30], i.e. $g_{1}(s)=g_{2}(s)=\ldots=$ $g_{N}(s)=\frac{1}{r_{\mathrm{b}}}$ in Figure 2, for some $r_{\mathrm{b}}>0$. The result that follows shows that for such networks one can explicitly compute the passivity margin $\sigma_{\mathrm{n}}$ that appears in Theorem 3 . Moreover, this margin is independent of the number of agents $N$ and becomes very large when the resistive connecting admittances are small.

Corollary 1. The electrical network shown in Figure 2, under Assumption 2, and considering the voltages $v_{1}, v_{2}, \ldots, v_{N}$ as inputs and the corresponding currents $i_{1}, i_{2}, \ldots, i_{N}$ as ouputs, with $g_{1}(s)=g_{2}(s)=\ldots=g_{N}(s)=\frac{1}{r_{\mathrm{b}}}$ and $r_{\mathrm{b}}>0$, is input-strictly passive with respect to $(\operatorname{Im} U, \operatorname{Im} U)$, when $U:=$ $\frac{1}{N} \overrightarrow{1} \overrightarrow{1}^{\prime}$, with passivity margin $\sigma_{\mathrm{n}}:=\frac{1}{2 r_{b}}>0$.

Proof of Corollary 1. Assume first that there is a proper admittance transfer function associated with the load. In this case, there exists a minimal state-space realization for the SISO LTI system representing the load admittance of the form

$$
\dot{x}_{\mathrm{L}}=A_{\mathrm{L}} x_{\mathrm{L}}+B_{\mathrm{L}} u_{\mathrm{L}}, \quad y_{\mathrm{L}}=C_{\mathrm{L}} x_{\mathrm{L}}+D_{\mathrm{L}} u_{\mathrm{L}},
$$

where $x_{\mathrm{L}} \in \mathbb{R}^{m}, u_{\mathrm{L}} \in \mathbb{R}$ is a voltage input, $y_{\mathrm{L}} \in \mathbb{R}$ is the corresponding current output. Using the Thevenin equivalent circuit [31] obtained by opening the branch corresponding to the load, one has that $u_{\mathrm{L}}(t)=v_{\mathrm{th}}(t)-r_{\mathrm{eq}} y_{\mathrm{L}}(t)$, where $r_{\mathrm{eq}}=\frac{r_{\mathrm{b}}}{N}$ is the Thevenin equivalent resistance, and $v_{\mathrm{th}}(t)=$ $\left(\frac{1}{N} \overrightarrow{1}^{\prime}\right) u_{\mathrm{n}}(t)$ is the resulting open-circuit voltage depending on the value of the vector of applied voltages $u_{\mathrm{n}}(t)=y_{\mathrm{a}}(t)$ to the electrical network. The corresponding outputs of the electrical network (i.e., the corresponding currents through the connecting branches) are given by $y_{k}^{\mathrm{n}}(t)=\frac{1}{r_{\mathrm{b}}}\left(u_{k}^{\mathrm{n}}(t)-u_{\mathrm{L}}(t)\right)$. From these considerations and identifying the state vector $x_{\mathrm{n}}$ of the electrical network with the state vector associated with the load $x_{\mathrm{L}}$, one obtains the following minimal realization (12) for the electrical network $G(s)$ :

$$
\begin{aligned}
A_{\mathrm{n}} & =A_{\mathrm{L}}-\left[r_{\mathrm{b}} /\left(N+r_{\mathrm{b}} D_{\mathrm{L}}\right)\right] B_{\mathrm{L}} C_{\mathrm{L}}, \\
B_{\mathrm{n}} & =\left[1 /\left(N+r_{\mathrm{b}} D_{\mathrm{L}}\right)\right] B_{\mathrm{L}} \overrightarrow{1}^{\prime}, \\
C_{\mathrm{n}} & =\left[1 /\left(N+r_{\mathrm{b}} D_{\mathrm{L}}\right)\right] \overrightarrow{1} C_{\mathrm{L}}, \\
D_{\mathrm{n}} & =\left(1 / r_{\mathrm{b}}\right)\left\{I_{N}-\left[1 /\left(N+r_{\mathrm{b}} D_{\mathrm{L}}\right)\right] \overrightarrow{1} \overrightarrow{1}^{\prime}\right\} .
\end{aligned}
$$

If instead of a proper admittance, there is a proper impedance transfer function associated with the load, then there still exists a minimal state-space realization (28) for the SISO LTI system representing the load, but $u_{\mathrm{L}}(t) \in \mathbb{R}$ is now a current input and $y_{\mathrm{L}}(t) \in \mathbb{R}$ the corresponding voltage output. By using the Norton equivalent circuit [31] obtained by short circuiting the branch corresponding to the load and following the same approach as before, one obtains the following minimal realization (12) for the electrical network $G(s)$ :

$$
\begin{aligned}
& A_{\mathrm{n}}=A_{\mathrm{L}}-\left[N /\left(r_{\mathrm{b}}+N D_{\mathrm{L}}\right)\right] B_{\mathrm{L}} C_{\mathrm{L}}, \\
& B_{\mathrm{n}}=\left[1 /\left(r_{\mathrm{b}}+N D_{\mathrm{L}}\right)\right] B_{\mathrm{L}} \overrightarrow{1}^{\prime}, \\
& C_{\mathrm{n}}=-\left[1 /\left(r_{\mathrm{b}}+N D_{\mathrm{L}}\right)\right] \overrightarrow{1} C_{\mathrm{L}}, \\
& D_{\mathrm{n}}=\left(1 / r_{\mathrm{b}}\right)\left\{I_{N}-\left[1 /\left(r_{\mathrm{b}}+N D_{\mathrm{L}}\right)\right] \overrightarrow{1}^{\prime} \overrightarrow{1}^{\prime}\right\} .
\end{aligned}
$$

On the other hand, by noticing that, for $M=I_{N}-U, \overrightarrow{1}^{\prime} M=$ $\overrightarrow{1}^{\prime}-\frac{1}{m} \overrightarrow{1^{\prime}} \overrightarrow{1} \overrightarrow{1}^{\prime}=0$, we conclude that for both (29) and (30) we have that $B_{\mathrm{n}} M=0, C_{\mathrm{n}}^{\prime} M=0, D_{\mathrm{n}} U=U D_{\mathrm{n}}$, and $D_{\mathrm{n}}^{\prime} M=$ $\frac{1}{r_{b}}\left(I_{N}-U\right)$. Therefore, the commuting conditions in (16) hold. Moreover, (8c) is equivalent to find $P=P_{\mathrm{n}}=P_{\mathrm{n}}^{\prime}>0$, such that

$$
\begin{aligned}
& T^{\prime} {\left[\begin{array}{ll}
\mathcal{Q}_{\mathrm{n}} & \mathcal{S}_{\mathrm{n}} \\
\mathcal{S}_{\mathrm{n}}^{\prime} & \mathcal{R}_{\mathrm{n}}
\end{array}\right] T \leqslant 0, } \\
& \mathcal{Q}_{\mathrm{n}}=P_{\mathrm{n}} A_{\mathrm{n}}+A_{\mathrm{n}}^{\prime} P_{\mathrm{n}} \leqslant 0, \\
& \mathcal{R}_{\mathrm{n}}=2\left[2 \sigma_{\mathrm{n}}-1 /\left(r_{\mathrm{b}}\right)\right]\left(I_{N}-U\right) \leqslant 0, \\
& \mathcal{S}_{\mathrm{n}}=0 .
\end{aligned}
$$

From Assumption 2, since the electrical network is an asymptotically stable LTI system ${ }^{7}$, we know that there exists a positive definite matrix $P_{\mathrm{n}}$ such that $P_{\mathrm{n}} A_{\mathrm{n}}+A_{\mathrm{n}}^{\prime} P_{\mathrm{n}}<0$. And since $\left(I_{N}-U\right)$ has an eigenvalue at 0 and the remaining equal to 1 , we conclude that $\left(I_{N}-U\right)$ is positive semi-definite. This means that (31) is feasible if, and only if, $\sigma_{\mathrm{n}} \leqslant \frac{1}{2 r_{b}}$.

\section{E. Special case: RLC agent dynamics}

Inspired by the dynamics of a (passive) RLC band-pass filter, we consider agents dynamics (11a) with

$$
\begin{array}{rlrl}
A_{\mathrm{a}} & =\left[\begin{array}{cc}
0 & \frac{1}{l_{\mathrm{a}}} \\
-\frac{1}{c_{\mathrm{a}}} & -\frac{1}{r_{\mathrm{a}} c_{\mathrm{a}}}
\end{array}\right], & B_{\mathrm{a}}=\left[\begin{array}{c}
0 \\
\frac{1}{c_{\mathrm{a}}}
\end{array}\right], \\
C_{\mathrm{a}}=\left[\begin{array}{ll}
0 & 1
\end{array}\right], & D_{\mathrm{a}}=0,
\end{array}
$$

and

$$
a(s)=\frac{s / c_{\mathrm{a}}}{s^{2}+\frac{\omega_{0}}{Q} s+\omega_{0}^{2}}, \quad \omega_{0}^{2}:=\frac{1}{l_{\mathrm{a}} c_{\mathrm{a}}}, \quad Q:=\frac{r_{\mathrm{a}}}{l_{\mathrm{a}} \omega_{0}} .
$$

Since $a(0)=0$, the only value $y_{\text {eq }} \in \mathbb{R}$ for which the overall system could have an equilibrium point for which all agents exhibit the same output must be $y_{\text {eq }}=0$ [cf. (27)]. Therefore, in applying the procedure outlined in Section III-C to select the nonlinearity $\phi$, we only need to make sure that $\frac{d \phi}{d y}(0) \in$ $\Gamma_{\text {unstable }} \cap\left(0, \sigma_{\mathrm{n}}+\sigma_{\mathrm{a}}\right)$.

The result that follows shows that the passivity margin of such agents is solely determined by the value of the resistance $r_{\mathrm{a}}$ and that one can independently select the passivity margin, the center frequency $\omega_{0}$, and the quality factor $Q$. A high

\footnotetext{
${ }^{7}$ Actually, it is possible to show that every such symmetric electrical network with resistive only branches is asymptotically stable, and Assumption 2 is automatically fulfilled.
} 
quality factor will result in low harmonic distortion for the agents' outputs, because each agent will filter out all but the input harmonic with frequency close to $\omega_{0}$ (cf. Figure 4).

Lemma 8. The agent subsystem (11a) with (32) is outputstrictly passive in the usual sense, i.e., it is output-strictly passive with respect to $(\{0\},\{0\})$, with passivity margin $\sigma_{\mathrm{a}}=$ $\frac{1}{2 r_{\mathrm{a}}}>0$. Moreover, one can select the RLC parameters to obtain arbitrary positive values for the passivity margin $\sigma_{\mathrm{a}}$, center frequency $\omega_{0}$, and quality factor $Q$ by setting:

$$
r_{\mathrm{a}}=\frac{1}{2 \sigma_{a}}, \quad l_{\mathrm{a}}=\frac{1}{2 Q \sigma_{\mathrm{a}} \omega_{0}}, \quad c_{\mathrm{a}}:=\frac{2 Q \sigma_{\mathrm{a}}}{\omega_{0}} .
$$

Proof of Lemma 8. For these agents, (8d) is equivalent to $P=$ $P_{\mathrm{a}}=P_{\mathrm{a}}^{\prime}>0, P_{\mathrm{a}} A_{\mathrm{a}}+A_{\mathrm{a}}^{\prime} P_{\mathrm{a}}+4 \sigma_{\mathrm{a}} C_{\mathrm{a}}^{\prime} C_{\mathrm{a}} \leqslant 0, P_{\mathrm{a}} B_{\mathrm{a}}=C_{\mathrm{a}}^{\prime}$, because $D_{\mathrm{a}}=0$. Since $P_{\mathrm{a}}=\left[\begin{array}{ll}p_{11} & p_{12} \\ p_{12} & p_{22}\end{array}\right]$, we conclude from $P_{\mathrm{a}} B_{\mathrm{a}}=C_{\mathrm{a}}^{\prime}$ that we must have $p_{12}=0$, and $p_{22}=c_{\mathrm{a}}$. In this case, $P_{\mathrm{a}} A_{\mathrm{a}}+A_{\mathrm{a}}^{\prime} P_{\mathrm{a}}+4 \sigma_{\mathrm{a}} C_{\mathrm{a}}^{\prime} C_{\mathrm{a}} \leqslant 0$ becomes

$$
\left[\begin{array}{cc}
0 & \frac{p_{11}}{l_{\mathrm{a}}}-1 \\
\frac{p_{11}}{l_{\mathrm{a}}}-1 & 4 \sigma_{\mathrm{a}}-\frac{2}{r_{\mathrm{a}}}
\end{array}\right] \leqslant 0 \Leftrightarrow p_{11}=l_{\mathrm{a}}, \sigma_{\mathrm{a}} \leqslant \frac{1}{2 r_{\mathrm{a}}},
$$

which confirms that the passivity margin is indeed $\frac{1}{2 r_{\mathrm{a}}}$. The formulas in (34) are then obtained by solving (33) for the desired values of $\omega_{0}$ and $Q$.

\section{NUMERICAL RESULTS}

In this section we provide numerical results for the interconnection network shown in Figure 6, with $N=3$ agents with RLC dynamics (see Section III-E). The following parameters were used for the agents: $\omega_{0}=2 \pi \times 60 \mathrm{rad} / \mathrm{s}$, $Q=1500, \sigma_{\mathrm{a}}=0.0025$, leading through (34) to $r_{\mathrm{a}}=200 \Omega$, $l_{\mathrm{a}}=0.530 \mathrm{mH}, c_{\mathrm{a}}=13.263 \mathrm{mF}$. Two cases were considered for the network: $($ i $) l_{\mathrm{b}}=0$, and (ii) $l_{\mathrm{b}}=1 \mathrm{mH}$. In both cases the following parameters were used: $r_{\mathrm{b}}=0.25 \Omega, R_{1}=3 \Omega$, $L_{1}=1 \mathrm{mH}, C_{1}=100 \mu \mathrm{F}, R_{2}=100 \Omega$.

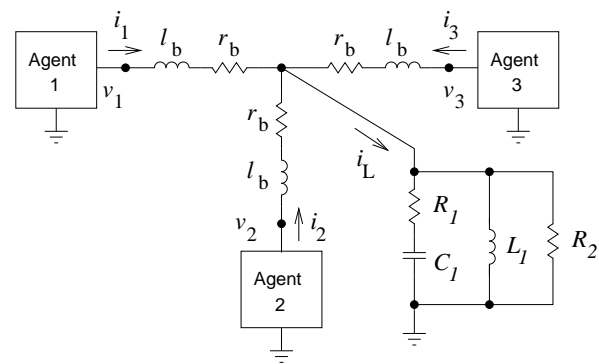

Figure 6. Three agents interconnected by means of a symmetric star-shaped electrical network to supply energy to the reactive load represented by $R_{1}$, $R_{2}, C_{1}$ and $L_{1}$.

For $l_{\mathrm{b}}=0$, in view of Corollary 1 , the network is inputstrictly passive with respect to $(\operatorname{Im} U, \operatorname{Im} U), U:=\frac{1}{N} \overrightarrow{1} \overrightarrow{1}^{\prime}$ with passivity margin $\sigma_{\mathrm{n}}:=\frac{1}{2 r_{b}}=2$. The associated root loci in (26) are depicted in Figure 7 and exhibit at least one unstable root for $\gamma \geqslant 0.17$. To maximize the instability of the pole at the origin, we selected a value for $\alpha$ just below the upper bound of $\sigma_{\mathrm{a}}+\sigma_{\mathrm{n}}=2.0025$, below which Theorem 3, condition C10, can guarantee synchronization. The nonlinearity $\phi$ was chosen to be a saturation function with slope at the origin equal $2 \alpha$ and breakpoints at \pm 15.59 . The values of these breakpoints are selected to obtain oscillations with amplitude of 120 volts. Figure 8 shows a simulation of the closed-loop for which the agents were initialized with very distinct initial conditions to emphasize the global nature of the convergence results. At steady state, the agents' outputs exhibit a Total Harmonic Distortion - THD of about $0.41 \%$

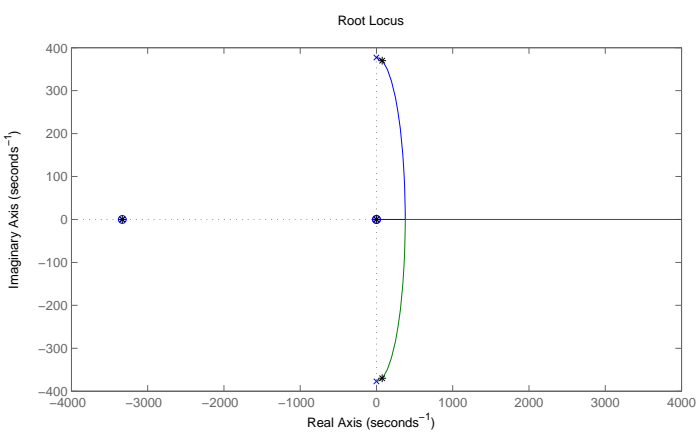

(a) root locus of $h_{\mathrm{L}}(s)$

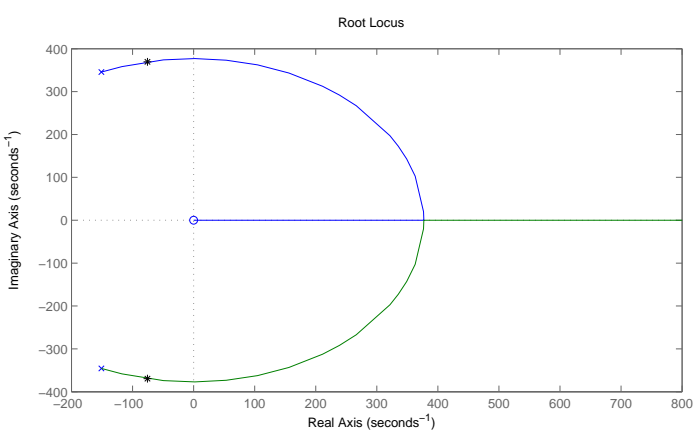

(b) root locus of $h_{\mathrm{b}}(s)$

Figure 7. Root-loci corresponding to the characteristic equations in (26). The "*" denotes the position of the poles for $\gamma=2.0025$ showing that $1-\gamma h_{\mathrm{L}}(s)=0$ has a pair of complex conjugate unstable roots, making this a suitable value for the parameter $\alpha$.

When $l_{\mathrm{b}}=1 \mathrm{mH}$, the electrical network is not input-strictly passive, and condition $\mathrm{C} 10$ in Theorem 3 cannot be applied anymore. To see this, notice that the direct application of input voltage signals, at $t=0$, will not result on instantaneous power dissipation in the resistances $r_{\mathrm{b}}$ if the currents in the interconnecting branches are initially zero. However, condition C11 still applies, because the electrical network is outputstrictly passive with respect to $(\operatorname{Im} U, \operatorname{Im} U)$, with passivity margin $\sigma_{\mathrm{n}}=0.125=\frac{r_{\mathrm{b}}}{2}>0$, as it can be verified by computing the LMI (8d). In addition, since the agent subsystem is output-strictly passive with respect to $(\{0\},\{0\})$ (c.f. Lemma 8), incidentally the agent subsystem is also inputfeedforward passive with respect to $(\{0\},\{0\})$ with passivity margin $\sigma_{\mathrm{a}}=0$. By performing the same root loci analysis in Lemma 7, but now considering the new interconnecting admittance $g_{\mathrm{b}}(s)=\left(l_{\mathrm{b}} s+r_{\mathrm{b}}\right)^{-1}$, one can show that there is at least one unstable root for $\gamma \geqslant \gamma_{\min }=0.1005$. From Theorem 


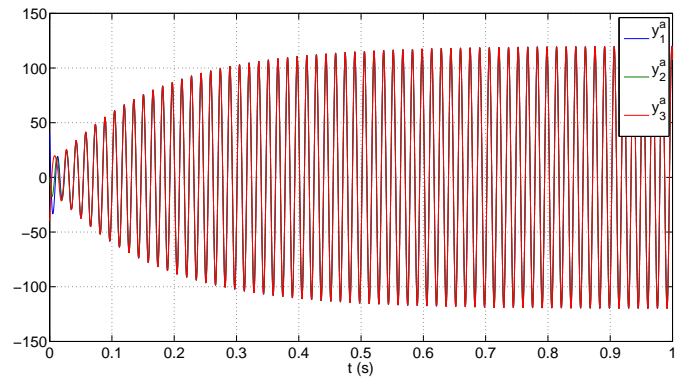

(a)

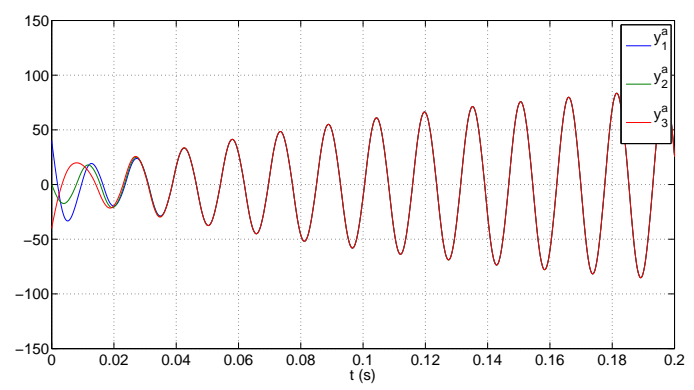

(b)

Figure 8. Simulated results for the 3-agent network shown in Figure 6, with $l_{\mathrm{b}}=0$. We can see in plot 8(a) that the voltage amplitudes take less than $0.8 \mathrm{sec}$ to reach their steady-state values. The plot $8(\mathrm{~b})$ is focused on the first $0.2 \mathrm{sec}$ of the same simulation, where we can see that the agents synchronize much faster, in less than $30 \mathrm{~ms}$.

3 , condition $\mathrm{C} 11$, synchronization of the inputs to the electrical network is guaranteed by choosing the nonlinearity $\phi$ as a saturation function with slope at the origin equal $\frac{1}{2 \alpha} \geqslant \gamma_{\min }$, with $\frac{1}{2 \alpha}>\frac{1}{\sigma_{n}}$. In addition, the feedback composition between agents' LTI subsystems and the electrical network, depicted in Figure 4 as $F(s)$, is asymptotically stable (this can be confirmed in the root loci analysis for $\gamma=0$ ). This fact together with the network symmetry and the synchronization of agents' inputs are sufficient to guarantee that the outputs of the agents will synchronize as shown in Figure 9. The saturation function breakpoints were adjusted to \pm 9.5 , and the slope $\frac{1}{2 \alpha}=10$, resulting in an amplitude of 120 volts in steady-state, with THD of less than $0.3 \%$.

\section{CONClusions AND DiRECTIONS FOR FUtURE RESEARCH}

We analyzed the synchronization of multiple identical nonlinear oscillators through a multiple-input/multiple output symmetric dynamical network and provide a constructive procedure for the design of the oscillators relying on sufficient conditions for output synchronization. These conditions are related to a newly introduced notion of passivity with respect to input/output manifolds.

Two key aspects distinguish this work from most of the previous research in this area: the coupling between oscillators persists even after they attain synchronization, and the interconnection structure is a dynamical system.

The conditions provided to establish synchronization are sufficient but not necessary, which leaves room for improving

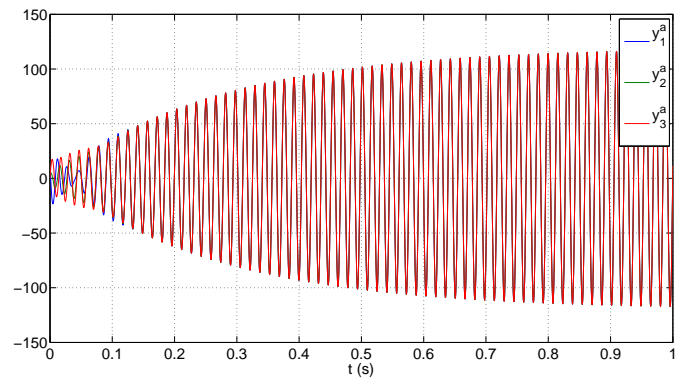

(a)

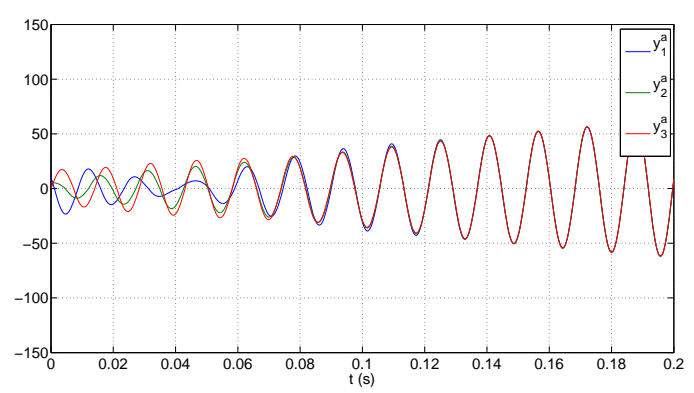

(b)

Figure 9. Simulated results for the 3-agent network shown in Figure 6, with $l_{\mathrm{b}}=1 \mathrm{mH}$. We can see in plot 9(a) that the voltage amplitudes take approximately $1 \mathrm{sec}$ to reach their steady-state values. The plot 9 (b) is focused on the first $0.2 \mathrm{sec}$ of the same simulation, where we can see that the agents synchronize much faster, in less than $140 \mathrm{~ms}$, but still slower than the case $l_{\mathrm{b}}=0$.

the results presented. In fact, simulation studies indicate that more relaxed conditions should be possible. On the other hand, at least for the special case of resistive-only interconnecting branches (Section III-D), the sufficient conditions do not depend on the number $N$ of interconnected agents.

While we establish that the inputs and outputs of the oscillators asymptotically synchronize with persistent oscillations, we do not provide explicit mechanisms to control the amplitude of the oscillations, or to verify that the oscillations will be almost sinusoidal signals. Since this is crucial for the design of electric power supplies, the constructive procedure presented here needs to be augmented with a mechanism to regulate the magnitude of the oscillations (the authors have some preliminary results in this direction [20, 21], but formal convergence proofs are still lacking), and to enforce a prescribed upper bound on the Total Harmonic Distortion (THD) associated with the signals generated by the nonlinear oscillators.

\section{ACKNOWLEDGMENTS}

The first author express thanks to the Universidade Federal de Minas Gerais, and to the Conselho Nacional de Desenvolvimento Cient'fico e Tecnológico - CNPq, Brazil, for financial support under grant 200312/2010-3. 


\section{APPENDIX}

Proposition 2. Given $N$ vectors $a_{1}, a_{2}, \ldots, a_{N} \in \mathbb{R}^{n}$ and $N$ scalars $\alpha_{1}, \alpha_{2}, \ldots, \alpha_{N}>0$,

$$
\left\|\sum_{k=1}^{N} a_{k}\right\|^{2} \leqslant\left(\sum_{j=1}^{N} \alpha_{j}^{-1}\right) \sum_{k=1}^{N} \alpha_{k}\left\|a_{k}\right\|^{2}
$$

Proof of Proposition 2. We prove this result by induction on $N$. The basis of induction $N=1$ holds with equality. To prove the induction step, we pick some $N>1$ and conclude from Young's inequality that for every $\epsilon>0$,

$$
\begin{aligned}
\left\|\sum_{k=1}^{N} a_{k}\right\|^{2} & \leqslant\left\|\sum_{k=1}^{N} a_{k}\right\|^{2}+\left\|a_{N}\right\|^{2}+2\left\|\sum_{k=1}^{N} a_{k}\right\|\left\|a_{N}\right\| \\
& \leqslant\left(1+\epsilon^{-1}\right)\left\|\sum_{k=1}^{N-1} a_{k}\right\|^{2}+(1+\epsilon)\left\|a_{N}\right\|^{2} .
\end{aligned}
$$

Using the induction hypothesis, we then obtain

$$
\begin{aligned}
\left\|\sum_{k=1}^{N} a_{k}\right\|^{2} \leqslant & \left(1+\epsilon^{-1}\right)\left(\sum_{j=1}^{N-1} \alpha_{j}^{-1}\right) \sum_{k=1}^{N-1} \alpha_{k}\left\|a_{k}\right\|^{2} \\
& +(1+\epsilon)\left\|a_{N}\right\|^{2} .
\end{aligned}
$$

The result follows from setting

$$
\begin{aligned}
\epsilon=\left(\sum_{j=1}^{N-1} \alpha_{j}^{-1}\right) \alpha_{N} & \Leftrightarrow 1+\epsilon=\left(\sum_{j=1}^{N} \alpha_{j}^{-1}\right) \alpha_{N} \\
& \Leftrightarrow\left(1+\epsilon^{-1}\right)\left(\sum_{j=1}^{N-1} \alpha_{j}^{-1}\right)=\sum_{j=1}^{N} \alpha_{j}^{-1}
\end{aligned}
$$

Proof of Lemma 4. Defining $\hat{g}(s)=\frac{g_{\mathrm{b}}(s)^{2}}{N g_{\mathrm{b}}(s)+g_{\mathrm{L}}(s)}$, from (14) one has that

$$
\begin{aligned}
\lambda_{k}(s) v_{k}= & G(s) v_{k}=\left[g_{\mathrm{b}}(s) I_{N}-\hat{g}(s) \overrightarrow{1} \overrightarrow{1}^{\prime}\right] v_{k} \\
& \Rightarrow \underbrace{\left[\frac{g_{\mathrm{b}}(s)-\lambda_{k}(s)}{\hat{g}(s)}\right]}_{\mu_{k}} v_{k}=\overrightarrow{1}^{\prime} \overrightarrow{1}^{\prime} v_{k},
\end{aligned}
$$

and therefore, $\lambda_{k}(s)=g_{\mathrm{b}}(s)-\mu_{k} \hat{g}(s)$, with the eigenvalues $\mu_{k}$ of the constant matrix $E=\overrightarrow{1} \overrightarrow{1}^{\prime}$ given by $\mu_{1}=N, \mu_{2}=0$, $\mu_{3}=0, \ldots, \mu_{N}=0$. In this case, $\lambda_{1}(s)=g_{\mathrm{b}}(s)-N \hat{g}(s)=$ $\lambda_{\mathrm{L}}(s)$, and $\lambda_{2}(s)=\lambda_{3}(s)=\ldots=\lambda_{N}(s)=g_{\mathrm{b}}(s)=\lambda_{\mathrm{b}}(s)$. Since $\lambda_{\mathrm{b}}(s)=g_{\mathrm{b}}(s)$ and $\lambda_{\mathrm{L}}(s)=\left(\frac{N}{g_{\mathrm{L}}(s)}+\frac{1}{g_{\mathrm{b}}(s)}\right)^{-1}$, it follows that both $\lambda_{\mathrm{L}}(s)$ and $\lambda_{\mathrm{b}}(s)$ are admittances of equivalent circuits, since $\lambda_{\mathrm{L}}(s)$ corresponds to the series connection of the admittance $g_{\mathrm{b}}(s)$ and an admittance $g_{\mathrm{L}}(s) / N$. From the assumption that the electrical network is comprised only by passive elements, it follows that $\lambda_{\mathrm{L}}(s)$ and $\lambda_{\mathrm{b}}(s)$ are positive real transfer functions [17]. In addition, from (14), one has that

$$
G(s) \overrightarrow{1}=\left(g_{\mathrm{b}}(s)-\frac{N g_{\mathrm{b}}(s)^{2}}{N g_{\mathrm{b}}(s)+g_{\mathrm{L}}(s)}\right) \overrightarrow{1}=\lambda_{\mathrm{L}}(s) \overrightarrow{1} .
$$

Proof of Lemma 5. First notice that $A(s)=a(s) I_{N} \Rightarrow$ $U A(s)=A(s) U$, i.e. system $A(s)$ commutes with the pair of matrices $U, U \in \mathbb{R}^{N \times N}$. This, together with the assumption that $U G(s)=G(s) U$, leads to

$$
\begin{aligned}
{[I+A(s) G(s)] U } & =U[I+A(s) G(s)], \\
U[I+A(s) G(s)]^{-1} & =[I+A(s) G(s)]^{-1} U,
\end{aligned}
$$

with the last step obtained by multiplying the left and right hand sides of the previous expression by $[I+A(s) G(s)]^{-1}$. Therefore, since $A(s) U=U A(s)$,

$$
\begin{aligned}
F(s) U & =[I+A(s) G(s)]^{-1} A(s) U, \\
& =U[I+A(s) G(s)]^{-1} A(s)=U F(s) .
\end{aligned}
$$

\section{REFERENCES}

[1] F. Katiraei and M. R. Iravani, "Power management strategies for a microgrid with multiple distributed generation units," IEEE Trans. on Power Systems, vol. 21, no. 4, pp. 1821-1831, 2006.

[2] J. M. Guerrero, J. C. Vásquez, J. Matas, M. Castilla, and L. G. de Vicuña, "Control strategy for flexible microgrid based on parallel line-interactive UPS systems," IEEE Trans. on Industrial Electronics, vol. 56, no. 3, pp. 726736, 2009.

[3] T. Kawabata and S. Higashino, "Parallel operation of voltage source inverters," IEEE Trans. on Industry Applications, vol. 24, no. 2, pp. 281-287, 1988.

[4] A. M. Stanković and T. Aydin, "Analysis of asymmetrical faults in power systems using dynamical phasors," IEEE Trans. on Power Systems, vol. 15, no. 3, pp. 1062-1068, 2000.

[5] J. Rocabert, A. Luna, F. Blaabjerg, and P. Rodriguez, "Control of power converters in AC microgrids," IEEE Trans. on Power Electronics, vol. 27, no. 11, pp. 47344749, 2012.

[6] S. Caliskan and P. Tabuada, "Compositional transient stability analysis of multimachine power networks," IEEE Transactions on Control of Network Systems, vol. 1, no. 1, pp. 4-14, 2014.

[7] A. Tuladhar, H. Jin, T. Unger, and K. Mauch, "Parallel operation of single phase inverter modules with no control interconnections," vol. 1. Applied Power Electronics Conference and Exposition - APEC '97 Conference Proceedings, feb. 1997, pp. 94-100.

[8] J. M. Guerrero, L. Hang, and J. Uceda, "Control of distributed uninterruptible power supply systems," IEEE Trans. on Industrial Electronics, vol. 55, no. 8, pp. 28452859, 2008.

[9] E. Coelho, P. Cortizo, and P. Garcia, "Small-signal stability for parallel-connected inverters in stand-alone AC supply systems," IEEE Trans. on Industry Applications, vol. 38, no. 2, pp. 533-542, 2002.

[10] J. W. Simpson-Porco, F. Dörfler, and F. Bullo, "Synchronization and power-sharing for droop-controlled inverters in islanded microgrids," vol. 49, no. 9, pp. 2603-2611, 2013.

[11] G.-B. Stan and R. Sepulchre, "Analysis of interconnected oscillators by dissipativity theory," IEEE Trans. on Automatic Control, vol. 52, no. 2, pp. 256-270, 2007. 
[12] M. Arcak, "Passivity as a design tool for group coordination," IEEE Trans. on Automatic Control, vol. 52, no. 8, pp. 1380-1390, 2007.

[13] A. O. Hamadeh, G.-B. V. Stan, and J. M. Gonçalves, "Constructive synchronization of networked feedback systems," in 49th IEEE Conference on Decision and Control, Atlanta, USA, December 2010, pp. 6710-6715.

[14] J. C. Willems, "Dissipative dynamical systems," European Journal of Control, vol. 13, pp. 134-151, 2007.

[15] A. Y. Pogromsky, A. L. Fradkov, and D. J. Hill, "Passivity based damping of power system oscillations," in Proceedings of the IEEE Conference on Decision and Control, vol. 4, 1996, pp. 3876-3881.

[16] R. Ortega, M. Galaz, A. Astolfi, Y. Sun, and T. Shen, "Transient stabilization of multimachine power systems with nontrivial transfer conductances," IEEE Trans. on Automatic Control, vol. 50, no. 1, pp. 60-75, 2005.

[17] B. D. O. Anderson and S. Vongpanitlerd, Network Analysis and Synthesis: A Modern Systems Theory Approach. Prentice-Hall, Inc., 1973.

[18] H. K. Khalil, Nonlinear Systems, 3rd ed. Englewood Cliffs, NJ: Prentice Hall, 2002.

[19] R. Olfati-Saber, J. A. Fax, and R. M. Murray, "Consensus and cooperation in networked multi-agent systems," Proc. of the IEEE, vol. 95, no. 1, pp. 215-233, 2007.

[20] L. A. B. Torres, J. P. Hespanha, and J. Moehlis, "Power supply synchronization without communication," in Proc. of the IEEE Power and Energy Society General Meeting, 2012.

[21] M. de Azevedo Ávila, L. A. B. Tôrres, and P. F. Seixas, "Incremental passivity based parallel operation of uninterruptible power supplies without communication: Towards a digital implementation," in Brazilian Power Electronics Conference (COBEP), 2013, October 2013, pp. 414-419.

[22] E. A. Coddington and N. Levinson, Theory of Ordinary Differential Equations. Krieger Pub Co, 1984.

[23] K. Josić, "Invariant manifolds and synchronization of coupled dynamical systems," Physical Review Letters, vol. 80, no. 14, pp. 3053-3056, 1998.

[24] A. Pavlov and L. Marconi, "Incremental passivity and output regulation," Systems \& Control Letters, vol. 57, pp. 400-409, 2008.

[25] J. P. LaSalle, The Stability of Dynamical Systems, ser. Regional Conference Series in Applied Mathematics. Society for Industrial and Applied Mathematics, 1976, no. 25.

[26] G.-B. Stan, "Global analysis and synthesis of oscillations: a dissipativity approach," Ph.D. dissertation, Université de Liège, 2005.

[27] J. Ginoux and C. Letellier, "Van der Pol and the history of relaxation oscillations: Toward the emergence of a concept," Chaos, vol. 22, no. 2, 2012.

[28] R. Fitzhugh, "Computation of impulse initiation and saltatory conduction in a myelinated nerve fiber," Biophysical journal, vol. 2, pp. 11-21, 1962.

[29] A. Micallef, M. Apap, C. Spiteri-Staines, J. Guerrero, and J. Vasquez, "Reactive power sharing and voltage harmonic distortion compensation of droop controlled single phase islanded microgrids," IEEE Trans. on Smart Grid, 2014, article in Press.

[30] R. M. S. Filho, P. F. Seixas, P. C. Cortizo, and G. Gateau, "Small-signal stability enhancement of communicationless parallel connected inverters," in Industrial Electronics, 2009. IECON '09. 35th Annual Conference of IEEE, 2009, pp. 863-870.

[31] A. Agarwal and J. H. Lang, Foundations of Analog and Digital Electronic Circuits. Morgan Kaufmann Publishers, Elsevier, 2005.

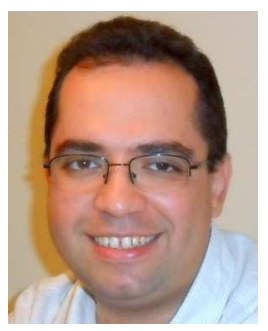

Leonardo A. B. Tôrres received his B.Eng. in 1997, and his PhD degree in 2001, both in Electrical Engineering, from the Federal University of Minas Gerais (UFMG), Brazil. In 2010-2011 he was a visiting scholar at University of California Santa Barbara. He currently is an Associate Professor with the Department of Electronic Engineering, at UFMG, working with control and synchronization of nonlinear dynamical systems.

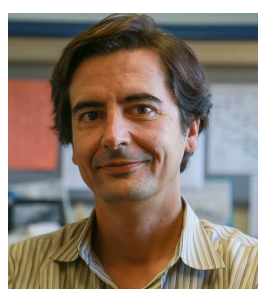

João P. Hespanha received his the Ph.D. degree in electrical engineering and applied science from Yale University, New Haven, Connecticut in 1998. From 1999 to 2001, he was Assistant Professor at the University of Southern California, Los Angeles. He moved to the University of California, Santa Barbara in 2002, where he currently holds a Professor position with the Department of Electrical and Computer Engineering. Prof. Hespanha is the Chair of the Department of Electrical and Computer Engineering and a member of the Executive Committee for the Institute for Collaborative Biotechnologies (ICB).

His current research interests include hybrid and switched systems; multiagent control systems; distributed control over communication networks (also known as networked control systems); the use of vision in feedback control; stochastic modeling in biology; and network security. Prof. Hespanha is the recipient of the Yale University's Henry Prentiss Becton Graduate Prize for exceptional achievement in research in Engineering and Applied Science, a National Science Foundation CAREER Award, the 2005 best paper award at the 2nd Int. Conf. on Intelligent Sensing and Information Processing, the 2005 Automatica Theory/Methodology best paper prize, the 2006 George S. Axelby Outstanding Paper Award, and the 2009 Ruberti Young Researcher Prize. Prof. Hespanha is a Fellow of the IEEE and an IEEE distinguished lecturer since 2007.

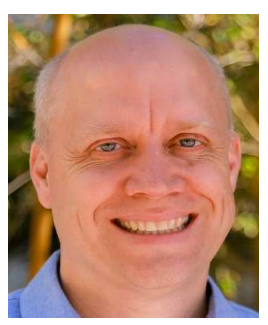

Jeff Moehlis received the B.S. degree in Physics and Mathematics from Iowa State University in 1993, and the Ph.D.degree in Physics from the University of California, Berkeley, in 2000. He was a Postdoctoral Researcher in the Program in Applied and Computational Mathematics at Princeton University from 2000 to 2003. In 2003, he joined the faculty of the Department of Mechanical Engineering at the University of California, Santa Barbara, where he is currently a professor with research interests in dynamical systems and control. He is the recipient of a Sloan Fellowship in Mathematics and a National Science Foundation CAREER Award. 